\title{
New candidate GHz peaked spectrum and compact steep spectrum sources
}

\author{
P. G. Edwards ${ }^{1}$ and S. J. Tingay ${ }^{2}$ \\ ${ }^{1}$ Institute of Space and Astronautical Science, Japan Aerospace Exploration Agency, Yoshinodai 3-1-1, Sagamihara, \\ Kanagawa 229-8510, Japan \\ e-mail: pge@vsop.isas.jaxa.jp \\ ${ }^{2}$ Centre for Astrophysics and Supercomputing, Swinburne University of Technology, PO Box 218, Hawthorn, Vic. 3122, \\ Australia \\ e-mail: stingay@astro.swin.edu.au
}

Received 26 November 2003 / Accepted 21 May 2004

\begin{abstract}
Data from a recent Australia Telescope Compact Array (ATCA) program of multi-frequency, multi-epoch monitoring of 202 active galactic nuclei with declinations $<+10^{\circ}$ have been searched for GHz Peaked Spectrum (GPS) and Compact Steep Spectrum (CSS) radio sources. Supplementary data at higher and lower frequencies, where available, have been used to further examine the spectral properties of previously reported and new candidate GPS and CSS sources. The ATCA monitoring program has allowed the variability and polarization properties of sources previously reported as GPS and CSS sources, and the majority of new GPS and CSS candidates, to be investigated, confirming that these are useful diagnostics in discriminating genuine GPS and CSS sources from variable sources that display similar spectra only temporarily. GPS sources are confirmed to be generally more compact, and less polarized, than CSS sources, although CSS sources show evidence for being somewhat less variable than GPS sources at 1.4 and $2.5 \mathrm{GHz}$. In addition, the widths of GPS spectra are examined, and a significant difference is found in the GPS sample of Snellen et al. (2000) between sources with compact double (CD) or compact symmetric object (CSO) morphologies and sources with other morphologies, in that CD and CSO sources have generally narrower spectra. Possible reasons for this difference are considered.
\end{abstract}

Key words. surveys - galaxies: active - radio continuum: galaxies

\section{Introduction}

The classifications of GHz Peaked Spectrum (GPS) and Compact Steep Spectrum (CSS) radio sources are based on their total flux density spectra. The conventional, though somewhat arbitrary, classification scheme assigns the GPS label to sources with spectra which peak above $500 \mathrm{MHz}$, and the CSS label to sources peaking at lower frequencies (if a turnover can be detected at all). In addition to the spectral properties, morphological criteria are required for bona fide members of these classes, with GPS sources generally less than $1 \mathrm{kpc}$ in projected linear size and CSS sources less than $20 \mathrm{kpc}$. An observed anti-correlation between turnover frequency and projected linear size (Fanti et al. 1990; O’Dea \& Baum 1997) has led to a model in which GPS sources evolve into CSS sources, and possibly then into FR-I and/or FR-II sources (Snellen et al. 2003).

Studies of the motion of the components in the Compact Symmetric Object (CSO) sub-class of GPS/CSS sources indicate that they have become active in the last $\sim 10^{3}$ years (Conway 2002). In some cases this appears to be the first instance of activity; in other sources there is evidence that the sources have been re-activated after a period of quiescence (e.g., Tingay et al. 2003a). There is substantial evidence that mergers or other disruptions to the galaxy core have produced this activity. GPS radio sources are identified with both quasars and galaxies, and while GPS quasars share many of the radio properties of GPS galaxies, it has been argued that they are probably not a single class of object unified by orientation (Snellen et al. 1999).

Early interest in GPS sources stemmed from the fact that a significant fraction were identified with high redshift sources. This resulted in searches for GPS sources deliberately excluding sources identified with galaxies (Gopal-Krishna et al. 1983; Spoelstra et al. 1985). More recently, the focus of attention has shifted to source evolution, as the youth of (at least the CSO sub-class of) GPS sources provides the opportunity to study radio sources in early stages of development. GPS galaxies also have the advantage that high linear resolution observations can be obtained of the central regions (e.g., Snellen et al. 2003; Tingay et al. 2003a).

The generally accepted criteria for identifying GPS sources are: a spectral turnover at $\mathrm{GHz}$ frequencies, with a spectral index $\left(S \propto v^{+\alpha}\right)$ at low frequencies of $\alpha>+0.5$ or a 
spectral index at high frequencies of $\alpha<-0.5$, and a spectral curvature of at least 0.6 (see Sect. 2). Sources with spectra that turn up at lower or higher frequencies were initially excluded from GPS catalogues (Gopal-Krishna et al. 1983), although it is becoming increasingly clear that this requirement may be too restrictive. In particular, the spectra of re-activated sources may have a turn up at lower frequencies corresponding to extended emission resulting from a previous active period. In addition to these criteria, GPS generally display at least some of the following properties: compact structure (confined to the central $1 \mathrm{kpc}$ ), usually resolved into two or three components by VLBI, low fractional radio polarization, low variability, and sub-luminal component motions. Compact Steep Spectrum sources share many of properties of GPS sources, with the main differences being, as mentioned above, that the spectral turnover, if present, occurs at lower frequencies, that CSS sources have higher fractional polarizations, and that the linear size is somewhat larger (see, e.g., O'Dea 1998).

In this paper we use data from a multi-frequency, multiepoch survey (Tingay et al. 2003b), undertaken with the Australia Telescope Compact Array (ATCA), to search for new GPS and CSS candidates. The survey, which was made in conjunction with the VSOP Survey Program (Hirabayashi et al. 2000), originally contained 202 sources, however 17 of these were dropped after the first few epochs as it was clear they did not meet the criteria for inclusion in the VSOP Survey Program. The remaining 185 sources were observed at up to 16 epochs between 1996 and 2000 at 1.384, 2.496, 4.800 and $8.640 \mathrm{GHz}$, with both total and polarized flux densities being measured. The multi-epoch observations allowed, for each source, an estimate of the variability index, $m$, defined as the rms variation from the mean flux density divided by the mean flux density. Sources were not imaged, but a flag was assigned based on the degree of compactness: "c" for compact sources, "e", extended, and "l", showing some sign of extension at low frequencies. The approximate angular resolution on the ATCA $6 \mathrm{~km}$ baseline is $7^{\prime \prime}$ at $1.4 \mathrm{GHz}, 4.5^{\prime \prime}$ at $2.5 \mathrm{GHz}$, $2^{\prime \prime}$ at $4.8 \mathrm{GHz}$, and $1^{\prime \prime}$ at $8.6 \mathrm{GHz}$.

In the tables in this paper we reproduce the values from Tingay et al. (2003b) for the source characteristics at $4.8 \mathrm{GHz}$, as this frequency band is commonly used to characterise sources (see, e.g., O'Dea 1998), and as it is close to the peak frequency for many of the GPS sources we consider. The mean variability indices at $4.8 \mathrm{GHz}$ for the 185 sources ranged between 0.01 and 0.43 , with a median value of 0.08 . The highest observed linear polarization was $8.09 \%$ at $4.8 \mathrm{GHz}$, with a median level of $2.21 \%$. Twenty-four sources (13\% of the 185 sources) had polarization levels below the reliably measurable level of $0.5 \%$.

In addition to the ATCA data, we have used data from RATAN-600 observations at 0.96, 2.30, 3.90, 7.70, 11.2, and $21.65 \mathrm{GHz}$ (Kovalev et al. 1999), VLA calibrator observations, particularly post-1998 $43 \mathrm{GHz}$ data, (http:// www.aoc.nrao.edu/ gtaylor/calib.html), ATCA calibrator observations, particularly at $22 \mathrm{GHz}$ (http:// www .narrabri.atnf.csiro.au/calibrators/), $843 \mathrm{MHz}$ SUMSS survey data (Mauch et al. 2003), archival $408 \mathrm{MHz}$ data from the MRC catalog (Large et al. 1981), archival
$365 \mathrm{MHz}$ data from Texas catalog (Douglas et al. 1996), and archival $160 \mathrm{MHz}$ data from the Culgoora catalog (Slee 1995). With the exception of the last three data sets, it has been attempted to use contemporaneous data as much as possible. The phenomenon of low frequency variability notwithstanding, use of the archival data at frequencies below $843 \mathrm{MHz}$ is acceptable as sources are expected to show less intrinsic variability at these frequencies. In several instances, other data have been used, and, in addition, a handful of $22 \mathrm{GHz}$ observations were made with the ATCA in April 2003 specifically for this study.

The ATCA monitoring was undertaken for sources south of a declination of $+10^{\circ}$, and so this study supplements the work of King et al. (1997) and Snellen et al. (2002) in redressing the under-representation of southern sources in GPS and CSS catalogs (see, e.g., O’Dea 1998).

In order to compare the characteristics of new candidate GPS and CSS sources with established members of these classes, we first examine the properties of previously reported sources before presenting the same properties for the new candidates. In Sect. 2 we study 20 previously reported GPS sources, and in Sect. 3 we present data for 8 new GPS candidates. In Sects. 4 and 5 we examine 8 previously reported, and 12 new candidate, CSS sources. In Sect. 6 we present the properties of a number of other sources of interest, which we find do not meet our criteria for inclusion as GPS or CSS candidates. After tabulating the properties of sources in each section, short comments are given for individual sources. These draw attention to particular properties of the source revealed by the ATCA monitoring, and are accompanied by illustrative, rather than exhaustive, notes from the literature.

\section{Previously reported GPS sources}

The properties of GPS sources have been comprehensively reviewed by O'Dea (1998). In Table 1 we list those known GPS sources which were included in the ATCA program, and plot their spectra in Fig. 1. The references given in Table 1 are to compilations of GPS sources which include the source in question, and generally, but not always, include the original report of discovery of the GPS-like nature of the source.

We also list in Table 1 the source classification and redshift, and the mean flux density, $S$, mean fractional polarization, $p$, and variability index, $m$, all at $4.8 \mathrm{GHz}$, from the ATCA monitoring data. Where possible, we also give for comparison, in brackets, the polarized flux measured with the VLA at $4.9 \mathrm{GHz}$ (Perley 1982). The spectral index at high frequency, $\alpha_{\text {hi }}$, is generally determined from the data at the highest frequencies plotted for that source in Figs. 1 and 2. The spectral curvature, $\Delta \alpha$, is the absolute difference between the high frequency and the low frequency spectral indices, following de Vries et al. (1997). The width is the $F W H M$ of the fitted spectrum in decades of frequency, following O'Dea et al. (1991). The flag is that from the ATCA data described in Sect. 1.

De Vries et al. (1997) have examined the spectra of GPS sources and derived an average spectrum with a optically thick spectral index of 0.5 below the peak, and a broken powerlaw spectrum above the peak changing from -0.4 to -0.7 at 
Table 1. Properties of previously identified GPS sources and candidates. Type Q denotes quasar; G, galaxy; GL, gravitational lens; and BL, BL Lac object. Other parameters are described in Sect. 2. The percentage polarized flux densities given in brackets are those measured at 4.9 GHz by Perley (1982).

\begin{tabular}{|c|c|c|c|c|c|c|c|c|c|c|c|c|c|}
\hline Source & $\begin{array}{l}\text { B1950 or } \\
\text { other }\end{array}$ & Refs. $^{a}$ & Type & $z$ & $\begin{array}{l}S_{4.8} \\
(\mathrm{Jy})\end{array}$ & $m_{4.8}$ & \multicolumn{2}{|c|}{$\begin{array}{l}p_{4.8} \\
(\%)\end{array}$} & $\begin{array}{c}v_{\mathrm{pk}} \\
(\mathrm{GHz})\end{array}$ & $\alpha_{\mathrm{hi}}$ & $\Delta \alpha$ & Width & Flag \\
\hline J0240-2309 & $0237-233$ & K97V97098 & $Q$ & 2.223 & 2.88 & 0.03 & 3.47 & $(4.2)$ & 1.3 & -1.1 & 1.6 & 1.2 & $\mathrm{c}$ \\
\hline J0241-0815 & NGC1052 & V97 & $\mathrm{G}$ & 0.004 & 2.47 & 0.09 & $<0.5$ & $(0.3)$ & 7.5 & -0.6 & 1.2 & 1.4 & $\mathrm{c}$ \\
\hline $\mathrm{J} 0414+0534$ & $4 C+05.19$ & Ka97 & GL & 2.639 & 0.68 & 0.03 & $<0.5$ & & 0.4 & -1.2 & 1.7 & 1.5 & 1 \\
\hline $\mathrm{J} 0459+0229$ & $0457+024$ & V97098 & Q & 2.384 & 1.43 & 0.06 & $<0.5$ & $(0.1)$ & 2.4 & -0.9 & 2.2 & 1.1 & $\mathrm{c}$ \\
\hline $\mathrm{J} 0503+0203$ & $0500+019$ & G83V97098 & Q & 0.583 & 2.05 & 0.02 & $<0.5$ & $(0.2)$ & 2.8 & -0.6 & 1.4 & 1.4 & $\mathrm{c}$ \\
\hline J0745-0044 & $0743-006$ & V97098 & Q & 0.994 & 2.05 & 0.04 & 0.61 & & 6.9 & -0.5 & 1.4 & 1.2 & $\mathrm{c}$ \\
\hline J0900-2808 & $0858-279$ & S85V97 & Q & 2.152 & 3.18 & 0.08 & 0.52 & & 5.2 & -1.0 & 1.5 & 1.2 & $\mathrm{c}$ \\
\hline J1146-2447 & $1143-245$ & V97098 & Q & 1.950 & 1.41 & 0.04 & 0.60 & (2.1) & 3.1 & -1.2 & 2.3 & 1.3 & $\mathrm{c}$ \\
\hline J1357-1744 & $1354-174$ & K97V97 & $\mathrm{Q}$ & 3.147 & 1.16 & 0.03 & 4.92 & & 1.9 & -0.6 & 1.0 & 1.7 & 1 \\
\hline $\mathrm{J} 1445+0958$ & $1442+101$ & V97098 & $\mathrm{Q}$ & 3.535 & 1.07 & 0.03 & 2.02 & (2.0) & 1.0 & -0.9 & 1.3 & 1.3 & $\mathrm{c}$ \\
\hline $\mathrm{J} 1522-2730$ & $1519-273$ & G83V97 & $\mathrm{BL}$ & 1.294 & 1.74 & 0.15 & 2.45 & (2.6) & 5.8 & -0.5 & 1.0 & 1.5 & 1 \\
\hline $\mathrm{J} 1543-0757$ & $1540-077$ & V00 & $\mathrm{G}$ & 0.172 & 1.00 & 0.02 & $<0.5$ & & 0.7 & -0.7 & 0.8 & 1.9 & $\mathrm{c}$ \\
\hline $\mathrm{J} 1723-6500$ & $1718-649$ & Т97 & $\mathrm{G}$ & 0.014 & 4.64 & 0.02 & $<0.5$ & & 2.7 & -0.6 & 1.1 & 1.8 & $\mathrm{c}$ \\
\hline $\mathrm{J} 1744-5144$ & $1740-517$ & K97 & $\mathrm{G}$ & $\ldots$ & 3.88 & 0.02 & $<0.5$ & & 1.0 & -0.8 & 1.0 & 1.7 & $\mathrm{c}$ \\
\hline J1939-6342 & $1934-638$ & K97V97 & G & 0.183 & 5.84 & 0.01 & $<0.5$ & & 1.4 & -1.2 & 2.2 & 1.0 & $\mathrm{c}$ \\
\hline J2003-3251 & $2000-330$ & V97 & $\mathrm{Q}$ & 3.773 & 0.92 & 0.04 & $<0.5$ & & 5.7 & -1.7 & 2.6 & 1.1 & $\mathrm{e}$ \\
\hline J2129-1538 & $2126-158$ & K97V97O98 & Q & 3.270 & 1.18 & 0.02 & $<0.5$ & $(0.4)$ & 6.9 & -0.6 & 1.5 & 1.3 & 1 \\
\hline $\mathrm{J} 2136+0041$ & $2134+004$ & O98 & Q & 1.936 & 9.47 & 0.02 & 0.60 & $(0.8)$ & 5.9 & -0.6 & 2.2 & 1.1 & $\mathrm{c}$ \\
\hline J2257-3627 & $2254-367$ & T03a & $\mathrm{G}$ & 0.006 & 1.28 & 0.04 & $<0.5$ & & 2.7 & -0.5 & 1.0 & 1.8 & $\mathrm{c}$ \\
\hline J2336-5236 & $2333-528$ & V97 & $\mathrm{G}$ & $\ldots$ & 1.63 & 0.02 & $<0.5$ & & 1.1 & -0.6 & 0.7 & 2.5 & 1 \\
\hline
\end{tabular}

${ }^{a}$ References: G83 = Gopal-Krishna et al. (1983); K97 = King et al. (1997); Ka97 = Katz et al. (1997); O98 = O’Dea (1998); S85 = Spoelstra et al. (1985); T97 = Tingay et al. (1997); T03a = Tingay et al. (2003a); V97 = de Vries et al. (1997); V00 = de Vries et al. (2000).

twice the peak frequency. However, here, for simplicity we have fitted a spectrum of the type

$\log S=a(\log v)^{2}+b \log v+c$

to the data for GPS sources (as also used, e.g., by Panajyan 1998). No physical meaning is ascribed to this fit: it is adopted only to estimate the peak frequency and the width of the spectrum in a consistent manner for all sources. It is clear from Fig. 1 that a spectrum of this form does not provide an accurate fit at frequencies far from the peak, and in some cases there are points which deviate from any simple spectral fit. As we are interested in the shape of the spectrum near the peak we have simply excluded these points in the spectral fit: the points neglected in this way are easily recognized in Figs. 1 and 2.

It is notable that the variability index, $m_{4.8}$, is less than or equal to the median value $(0.08)$ for 18 of the 20 sources. In addition, the fractional polarization, $p_{4.8}$, is less than median value for 17 of the 20 sources. Indeed, half of the 24 sources for which only an upper limit could be set to the fractional polarization are found in this table. Furthermore, all seven sources identified with galaxies have $p_{4.8}<0.5$. The one source that exceeds the median in both cases is the BL Lac object J1522-2730, which we consider in more detail below. Comparison of the structure flags is also interesting: of the 202 sources in the sample of Tingay et al. (2003b), about $50 \%$ had a "c" flag, $30 \%$ an "e" flag, and $20 \%$ an "l" flag. In contrast, of the 20 sources in Table 1, 14 have "c" flags, one has an "e" flag, and 5 have "l" flags. These results lend confidence to the use of these parameters in selecting GPS sources.

\subsection{Notes on individual sources}

In this section we add supplementary notes or comments on individual sources:

J0240-2309 has the second highest fractional polarization of this group. VLBI imaging reveals a core-jet structure with the compact core dominated at 2 and $5 \mathrm{GHz}$ by a steeper spectrum jet extending to the north-east (Fey et al. 1996; Fomalont et al. 2000).

J0241-0815 (0238-084, NGC1052) is a low redshift galaxy, considered in detail by Kameno et al. (2001) and Vermeulen et al. (2003). A spectrum compiled from an extensive list of sources is given by Tornikoski et al. (2000), revealing variability by a factor of $\sim 2$ over a wide range of frequencies. This variable nature is confirmed by the fact that the source has the second highest variability index in Table 1. The low frequency points lie above the nominal fit to the spectrum, and are qualitatively consistent with the notion that this source has previously been active, with the GPS spectrum reflecting a new stage of activity (e.g., Tingay et al. 2003a).

$\mathrm{J} 0414+0534(4 \mathrm{C}+05.19)$ is a gravitational lens system. The $178 \mathrm{MHz}$ data point is from the 4C catalog (Gower et al. 1967). A low variability index results from the ATCA monitoring, but archival data shows significant variation, ranging between 1.7 and $2.7 \mathrm{Jy}$, at $1.4 \mathrm{GHz}$. Some of this variability may be due to micro-lensing, which has been suggested as an explanation for the discrepant radio and optical flux ratios of the components 

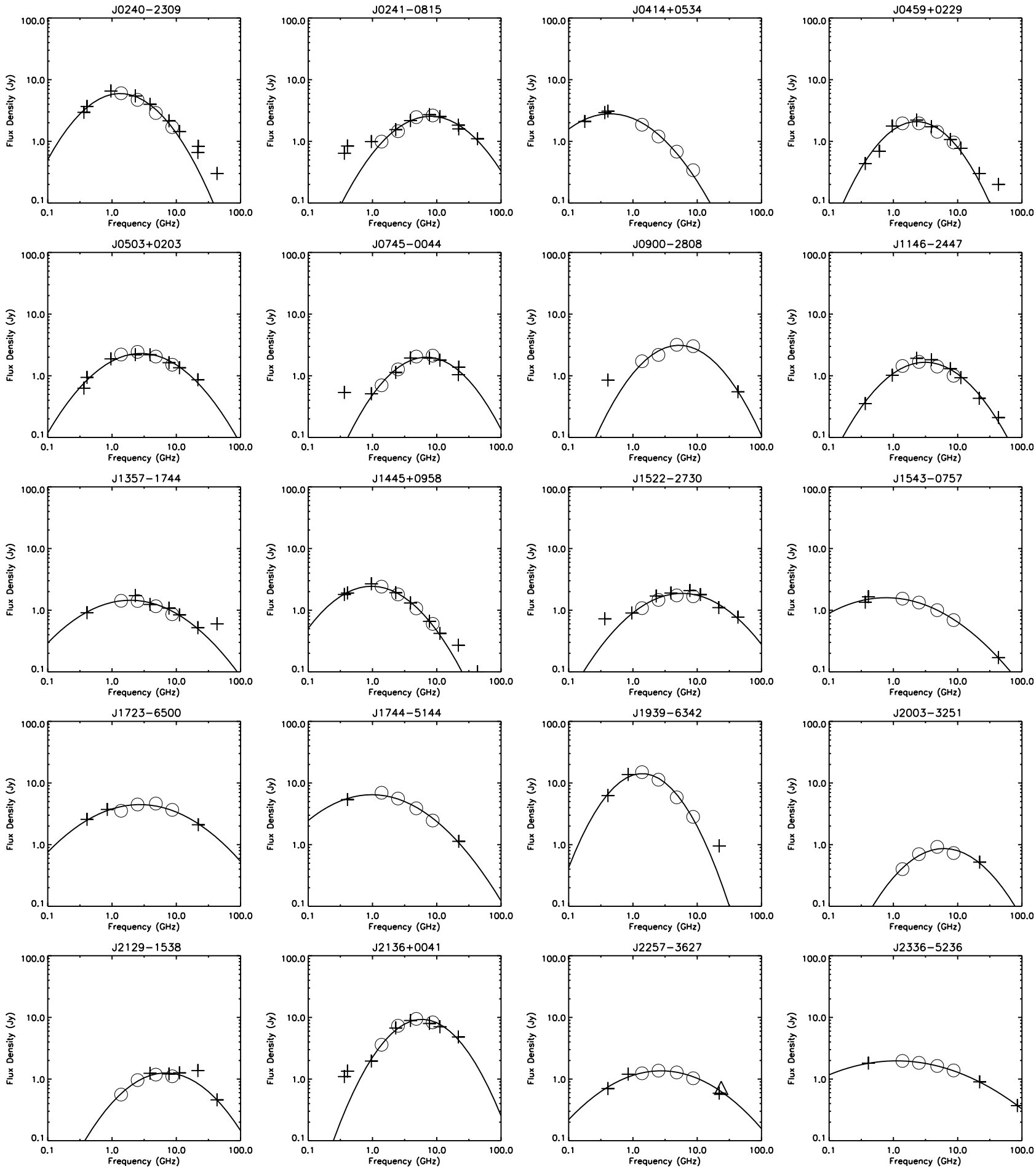

Fig. 1. Spectra of previously identified GPS sources. Circles denote data from Tingay et al. (2003b) and plus symbols denote data from the other sources described in Sect. 1. The triangles denote $22 \mathrm{GHz}$ observations made with the ATCA in April 2003 as part of this study. Any additional sources are listed in the Notes on individual sources. The fit to the spectra is described in Sect. 2 and fit parameters are given in Table 1.

(Witt et al. 1995). The peak of our fitted spectrum is $0.4 \mathrm{GHz}$, just below the nominal GPS/CSS division.

J0459+0229: the $606 \mathrm{MHz}$ point is from Kühr et al. (1981). VLBI images reveal a core with a jet directed to the north (e.g., Fey \& Charlot 2000; Fomalont et al. 2000).
J0503+0203: VLBI images of this CSO are given by Fey \& Charlot (2000), Fomalont et al. (2000) and Stanghellini et al. (2001).

J0745-0044 shows a pronounced turn up at low frequencies with a flux density at $178 \mathrm{MHz}$ of $2.7( \pm 15 \%)$ Jy (Gower et al. 1967). The spectral index at high frequencies is also flatter than 

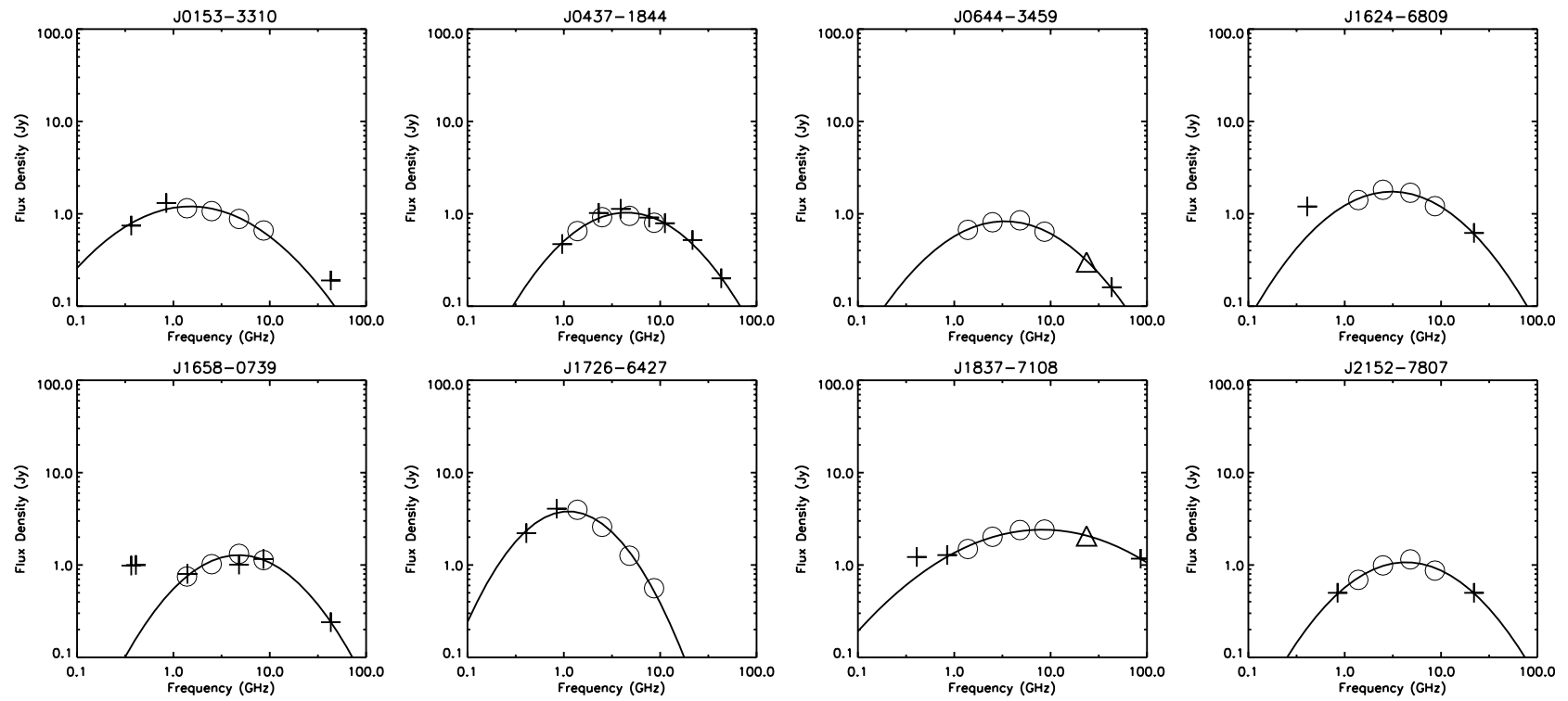

Fig. 2. Spectra of newly identified GPS sources. Symbols are the same as for Fig. 1. The fit to the spectra is described in Sect. 2 and results given in Table 2.

Table 2. Newly identified GPS sources and candidates. Type EF denotes empty field. Other types and parameters are as for Table 1.

\begin{tabular}{lccccccccccccc}
\hline \hline Source & B1950 & Type & $z$ & $\begin{array}{l}S_{4.8} \\
(\mathrm{Jy})\end{array}$ & $\begin{array}{l}m_{4.8} \\
\end{array}$ & $\begin{array}{c}p_{4.8} \\
(\%)\end{array}$ & $\begin{array}{l}v_{p k} \\
(\mathrm{GHz})\end{array}$ & $\alpha_{\mathrm{hi}}$ & $\Delta \alpha$ & Width & Flag \\
\hline $\mathrm{J} 0153-3310$ & $0150-334$ & $\mathrm{Q}$ & 0.610 & 0.88 & 0.04 & 1.35 & $(1.1)$ & 1.5 & -0.7 & 1.2 & 1.6 & $\mathrm{e}$ \\
$\mathrm{J} 0437-1844$ & $0434-188$ & $\mathrm{Q}$ & 2.702 & 0.95 & 0.05 & 0.80 & $(0.8)$ & 4.5 & -1.2 & 2.1 & 1.3 & 1 \\
$\mathrm{~J} 0644-3459$ & $0642-349$ & $\mathrm{Q}$ & 2.165 & 0.85 & 0.09 & 2.03 & $(1.1)$ & 3.3 & -1.0 & 1.3 & 1.4 & $\mathrm{c}$ \\
$\mathrm{J} 1624-6809$ & $1619-680$ & $\mathrm{Q}$ & 1.360 & 1.69 & 0.04 & $<0.5$ & & 3.1 & -0.7 & 1.1 & 1.4 & $\mathrm{c}$ \\
$\mathrm{J} 1658-0739$ & $1656-075$ & $\mathrm{EF}$ & $\ldots$ & 1.32 & 0.03 & $<0.5$ & & 4.8 & -1.0 & 1.5 & 1.2 & $\mathrm{e}$ \\
$\mathrm{J} 1726-6427$ & $1722-644$ & $\mathrm{EF}$ & $\ldots$ & 1.26 & 0.02 & $<0.5$ & & 1.1 & -1.4 & 2.2 & 1.0 & $\mathrm{c}$ \\
$\mathrm{J} 1837-7108$ & $1831-711$ & $\mathrm{Q}$ & 1.356 & 2.39 & 0.06 & 1.77 & & 8.2 & -0.4 & 0.8 & 2.0 & $\mathrm{c}$ \\
$\mathrm{J} 2152-7807$ & $2146-783$ & $\mathrm{Q}$ & $\ldots$ & 1.15 & 0.03 & $<0.5$ & & 4.3 & -0.6 & 1.2 & 1.3 & 1 \\
\hline
\end{tabular}

most GPS sources. A spectrum compiled from a much more extensive archival search is given in Tornikoski et al. (2000). A core dominated parsec-scale morphology is shown in the VLBA (pre-launch survey, VLBApls) image of Fomalont et al. (2000).

J0900-2808: the $80 \mathrm{MHz}$ flux density of $7 \mathrm{Jy}$ (Slee 1995) indicates a turn-up at low frequencies. The source is resolved out on $100 \mathrm{M} \lambda$ baselines in the $4.8 \mathrm{GHz}$ VLBA snapshot observation of Fomalont et al. (2000).

J1146-2447: the $2.1 \%$ polarized flux reported by Perley (1982) is significantly higher than the value of $0.6 \%$ from the ATCA monitoring. The VLBA observations of Fomalont et al. (2000) suggest a core-jet morphology.

J1357-1744 has the highest percentage linear polarization of this group. The $43 \mathrm{GHz}$ point suggests either a turn-up at high frequencies (possibly associated with the emergence of the core at these frequencies), or of variability. At $4.8 \mathrm{GHz}$, the compact parsec scale morphology was best fit by a bright central component with a fainter component $\sim 1$ mas away on either side (Fomalont et al. 2000).
J1445+0958: the $4.8 \mathrm{GHz}$ VLBA image of Fomalont et al. (2000) shows a core and bent parsec-scale jet.

J1522-2730 is classified as a BL Lac object, and, as noted in Sect. 2, shows the highest variability index of this group. The redshift for this object was recently reported by Heidt et al. (2004). Intra-Day Variability at radio wavelengths has been observed in this source (Kedziora-Chudczer et al. 2001; Jauncey et al. 2003) and VLBI imaging confirms the core is very compact (e.g., Fomalont et al. 2000). Tornikoski et al. (2001) present a composite spectrum derived from a large number of observations, and note that the source appears to have a relatively flat quiescent spectrum that is inverted during flares. The high variability and apparent lack of a persistent $\mathrm{GHz}$ peaked spectrum suggest this is less likely to be a bona fide GPS source.

J1543-0757: the VLBA image reveals two extended components separated by 50 mas, with the northern component $\sim 3$ three times brighter (Fomalont et al. 2000).

J1744-5144: VLBI observations at $2.3 \mathrm{GHz}$ reveal a widelyspaced, extended double with a separation of 52 mas (Jauncey et al. 2003). 
J1723-6500 is a low-redshift galaxy, considered in detail by Tingay et al. (1997, 2002, 2003a). A composite spectrum extending beyond $100 \mathrm{GHz}$ is given by Tornikoski et al. (2001). A detailed multi-frequency, multi-epoch study of the spectrum was undertaken more recently by Tingay \& de Kool (2003).

J1939-6342 is one of the earliest known GPS sources (Bolton et al. 1963; Kellermann 1966). The spectrum has the narrowest width of this group (which is discussed further in Sect. 7.2). VLBI imaging reveals the source has a compact double morphology with a separation of 42 mas (Tzioumis et al. 1989, 1998).

J2003-3251 is the highest-redshift source in this group, and has a probable core-jet morphology on VLBI scales (Fomalont et al. 2000).

J2129-1538: the Kovalev et al. (1999) $22 \mathrm{GHz}$ flux density of $1.374 \pm 0.021 \mathrm{Jy}$ does not lie on the spectrum defined by the other points. This may imply some variability at higher frequencies, which is also suggested by the spectrum compiled by Tornikoski et al. (2001). The source has a core dominated parsec-scale morphology (Fomalont et al. 2000).

J2136+0041 (OX 057): another source recognised early on to be a GPS source (Shimmins et al. 1968). The low frequency points lie above (and were not used in deriving) the fitted spectrum, a trait evident in the spectrum presented by Kraus et al. (1968). This is also apparent in the spectrum compiled by Tornikoski et al. (2001), which reveals that while the source shows some variability, the GPS character of the spectrum remains clearly present. Aller et al. (2002) present the centimetre wavelength variability over a 30 year period, which reveals modest long-term variability. Lovell et al. (2000) describe VSOP space VLBI observations of the source. Lister et al. (2002) note the source had been thought to be a symmetric double but that in fact it has a core-jet morphology.

J2257-3627 was identified as a GPS source in this ATCA monitoring program (Tingay et al. 2003a). As a low-redshift galaxy, high linear resolution studies over a range of wavelengths have been possible, and, as discussed in Tingay et al. (2003a), there is strong evidence for merger activity in the galaxy which is quite likely to be related to the GPS spectrum.

J2336-5236 has the broadest spectrum and smallest spectral curvature of the sources in this table. The source was originally identified as a quasar, however this was called into doubt (Jauncey et al. 1989), and more recently it has been identified as a galaxy (de Vries et al. 1995).

\section{Newly identified GPS sources and candidates}

In Table 2 the properties of eight new candidate GPS sources are presented, with the spectra plotted in Fig. 2. The primary requirements for selection were a spectral peak above $500 \mathrm{MHz}$, a spectral index at high frequencies of $\alpha_{\mathrm{hi}}<-0.5$, and a spectral curvature of at least 0.6 . As we found that the variability indices and fractional polarizations were lower for previously reported GPS sources, we were also guided in our selection by these parameters.

\subsection{Notes on individual sources}

J0153-3310 has a low variability index in the ATCA monitoring, but archival data suggest it is moderately variable on longer timescales, with $5 \mathrm{GHz}$ flux densities ranging from $0.860 \mathrm{Jy}$ in the PKS Catalogue (PKSCAT) (Wright \& Otrupcek 1990) to $1.378 \mathrm{Jy}$ in the PMN survey (Wright et al. 1996). The VLBA snapshot image of Fomalont et al. (2000) shows a coredominated structure that was best modelled by two components of similar flux density separated by 1 mas.

J0437-1844: VLBI imaging has been undertaken by Fey \& Charlot (2000) and Fomalont et al. (2000): the latter observation yielded a core-dominated structure modelled by two components of comparable flux density separated by 1 mas.

J0644-3459: the $4.8 \mathrm{GHz}$ VLBA image of Fomalont et al. (2000) is comprised of a core and a more extended (jet) component 3 mas to the west.

J1624-6809: including the $408 \mathrm{MHz}$ point in the fit to the spectrum would increase the FWHM from 1.4 to 1.7 . The $843 \mathrm{MHz}$ SUMSS flux density, when available, will be helpful in determining whether the spectrum is indeed intrinsically broader, or whether it is narrower but with a turn-up at lower frequencies. Beasley et al. (1997) give $5 \sigma$ upper limits at $89 \mathrm{GHz}$ and $147 \mathrm{GHz}$ of $0.08 \mathrm{Jy}$ and $0.05 \mathrm{Jy}$, respectively.

J1658-0739: the ATCA and VLA data points clearly yield a peaked spectrum, and the few archival flux density measurements confirm the source is not very variable. The 365 and $408 \mathrm{MHz}$ data points indicate a spectral turn-up at lower frequencies, suggestive of extended emission, and consistent with the "e" flag for this source. The low variability and polarization properties from the ATCA monitoring match those of canonical GPS sources very closely. The $4.8 \mathrm{GHz}$ VLBA image of Fomalont et al. (2000) is dominated by the core, with a weak secondary 7 mas away.

J1726-6427 has the narrowest spectrum of the sources in Table 2, similar to that of PKS 1934-638 (see Sect. 7.2), but otherwise currently little is known about this source.

J1837-7108: the spectrum is relatively broad, with a resulting spectral index between 22 and $89 \mathrm{GHz}$ of -0.4 , however the $146 \mathrm{GHz}$ flux density of $0.73 \mathrm{Jy}$ (Beasley et al. 1997) indicates the spectrum steepens, with a spectral index of -0.95 between 89 and $146 \mathrm{GHz}$. The PKSCAT flux density of $1.15 \mathrm{Jy}$ at $5.0 \mathrm{GHz}$ (Wright \& Otrupcek 1990) is roughly half that of the corresponding values from the PMN survey, 2.29 Jy (Wright et al. 1994), and the ATCA monitoring, 2.39 Jy. Similarly, the PKSCAT flux density at $2.7 \mathrm{GHz}$ of $1.32 \mathrm{Jy}$ is $\sim 65 \%$ of the $2.02 \mathrm{Jy}$ in the ATCA monitoring, suggesting the source has brightened significantly over the intervening period.

J2152-7807: identified as a quasar by Jauncey et al. (1989). Comparison with catalogued flux densities implies the source is moderately variable on long timescales. The PMN flux density of $1.128 \mathrm{Jy}$ (Wright et al. 1994) is in good agreement with the ATCA monitoring value of $1.15 \mathrm{Jy}$, with both being significantly in excess of the PKSCAT flux density of $0.77 \mathrm{Jy}$ 

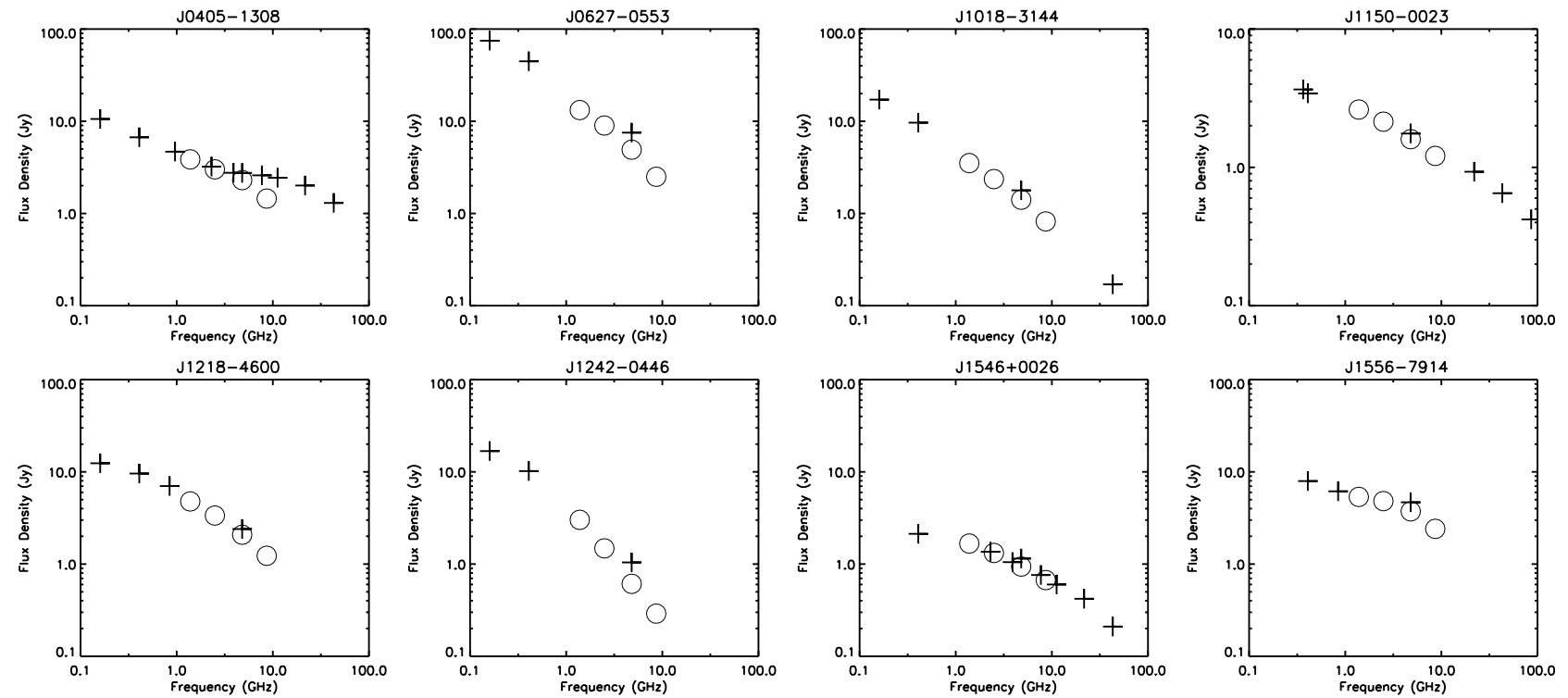

Fig. 3. Spectra of previously identified CSS sources. Symbols are the same as for Fig. 1.

Table 3. Properties of previously identified CSS sources and candidates. Parameters are the same as for Tables 1 and 2.

\begin{tabular}{|c|c|c|c|c|c|c|c|c|c|c|}
\hline Source & B1950 & Refs. ${ }^{a}$ & Type & $z$ & $\begin{array}{l}S_{4.8} \\
(\mathrm{Jy})\end{array}$ & $m_{4.8}$ & $\begin{array}{l}p_{4} \\
(\%\end{array}$ & & $\alpha$ & Flag \\
\hline J0405-1308 & $0403-132$ & K97 & $\mathrm{Q}$ & 0.571 & 2.30 & 0.05 & 2.40 & & -0.79 & $\mathrm{e}$ \\
\hline J0627-0553 & $0624-058$ & K97 & $\mathrm{EF}$ & $\ldots$ & 4.92 & 0.03 & 6.53 & & -1.15 & $\mathrm{e}$ \\
\hline J1018-3144 & $1015-314$ & K97 & $\mathrm{Q}$ & 1.246 & 1.41 & 0.02 & $<0.5$ & $(0.1)$ & -0.92 & $\mathrm{c}$ \\
\hline $\mathrm{J} 1150-0023$ & $1148-001$ & K97 & Q & 1.983 & 1.60 & 0.03 & 2.88 & & -0.53 & 1 \\
\hline $\mathrm{J} 1218-4600$ & $1215-457$ & K97 & $\mathrm{Q}$ & 0.529 & 2.08 & 0.03 & 2.23 & & -0.88 & $\mathrm{e}$ \\
\hline J1242-0446 & $1239-044$ & M92 & $\mathrm{G}$ & 0.480 & 0.61 & $\ldots$ & $\ldots$ & & -1.20 & $\mathrm{e}$ \\
\hline $\mathrm{J} 1546+0026$ & $1543+005$ & G83 & $\mathrm{G}$ & 0.550 & 0.94 & 0.05 & $<0.5$ & & -0.59 & 1 \\
\hline J1556-7914 & $1549-790$ & K97 & $\mathrm{G}$ & 0.150 & 3.73 & 0.02 & $<0.5$ & & -0.74 & $\mathrm{c}$ \\
\hline
\end{tabular}

$\bar{a}$ References: G83 = Gopal-Krishna et al. (1983); K97 = King et al. (1997); M92 = Mantovani et al. (1992).

(Wright \& Otrupcek 1990). Beasley et al. (1997) give $5 \sigma$ upper limits at $3 \mathrm{~mm}(89 \mathrm{GHz})$ and $2 \mathrm{~mm}(147 \mathrm{GHz})$ from SEST observations of 0.06 and $0.11 \mathrm{Jy}$, respectively.

\section{Previously identified CSS sources and candidates}

The properties of previously reported CSS sources are listed in Table 3 and their spectra are plotted in Fig. 3. The spectral indices, $\alpha$, given in Tables 3 and 4 are determined between 4.8 and $8.4 \mathrm{GHz}$ from the ATCA monitoring (Tingay et al. 2003b).

It is notable that, in contrast to the GPS sources and candidates in Tables 1 and 2, a number of the CSS sources have "e" flags, indicating evidence of extended structure on $6 \mathrm{~km}$ ATCA baselines. This is not unexpected, as the angular resolution at $4.8 \mathrm{GHz}$ on this baseline is $2^{\prime \prime}$, which corresponds to a linear resolution of about $10 \mathrm{kpc}$ for a source at $z \sim 0.5$. These previously reported CSS sources have, like the previously reported GPS sources, low variability indices.

\subsection{Notes on individual sources}

J0405-1308: the $80 \mathrm{MHz}$ flux density of Slee (1995) is $11 \mathrm{Jy}$, indicating that any turnover, if one exists, lies at lower frequencies. The higher flux density points from Kovalev et al. (1999), together with higher frequency points in the VLA catalog, suggest a flattening, or variability, of the spectrum above $\sim 6 \mathrm{GHz}$. The snapshot VLBA image of Fomalont et al. (2000) contains a very compact core and fainter jet extending to the south-east.

J0627-0553 (3C161): the $80 \mathrm{MHz}$ flux density of Slee (1995) is $111 \mathrm{Jy}$. Perley (1982) notes significant extended structure on VLA baselines, and the source was not detected in the VLBA observations of Fomalont et al. (2000).

J1018-3144: the source was highly resolved in the VLBA snapshot observation of Fomalont et al. (2000), with a correlated flux density of $0.5 \mathrm{Jy}$ detected only on the shortest baselines.

J1150-0023 (PMN J1150-0024): referred to as a GPS source by Jauncey et al. (2003), however it would appear any peak lies 
Table 4. Newly identified CSS sources and candidates. Parameters are the same as for previous Tables.

\begin{tabular}{|c|c|c|c|c|c|c|c|c|c|}
\hline Source & B1950 & Type & $z$ & $\begin{array}{l}S_{4.8} \\
(\mathrm{Jy})\end{array}$ & $m_{4.8}$ & \multicolumn{2}{|c|}{$\begin{array}{l}p_{4.8} \\
(\%)\end{array}$} & $\alpha$ & Flag \\
\hline J0059+0006 & $0056-001$ & Q & 0.719 & 1.37 & 0.02 & 7.25 & (5.8) & -0.52 & $\mathrm{c}$ \\
\hline J0119+0829 & $0116+082$ & G & 0.594 & 1.15 & 0.02 & 1.60 & & -0.72 & $\mathrm{c}$ \\
\hline J0155-4048 & $0153-410$ & G & 0.226 & 1.13 & 0.01 & 0.82 & & -0.63 & $\mathrm{c}$ \\
\hline J0616-3456 & 0614-349 & $\mathrm{G}$ & 0.329 & 1.33 & 0.02 & 0.61 & & -0.93 & $\mathrm{c}$ \\
\hline $\mathrm{J} 1351-1449$ & $1349-145$ & $\mathrm{EF}$ & $\ldots$ & 0.75 & 0.05 & $<0.5$ & & -0.67 & $\mathrm{c}$ \\
\hline J1424-4913 & $1421-490$ & $\mathrm{EF}$ & $\ldots$ & 5.06 & 0.03 & 3.22 & & -0.61 & $\mathrm{e}$ \\
\hline $\mathrm{J} 1435-4821$ & $1431-481$ & $\mathrm{EF}$ & $\ldots$ & 1.03 & 0.02 & 1.01 & & -0.81 & $\mathrm{c}$ \\
\hline J1501-3918 & $1458-391$ & $\mathrm{EF}$ & $\ldots$ & 1.17 & 0.02 & $<0.5$ & & -0.97 & $\mathrm{c}$ \\
\hline $\mathrm{J} 1526-1351$ & $1524-136$ & Q & 1.687 & 1.20 & 0.02 & 2.56 & (2.8) & -0.80 & $\mathrm{e}$ \\
\hline J1940-6908 & $1935-692$ & Q & 3.154 & 0.90 & 0.09 & 2.01 & & -0.49 & 1 \\
\hline J2206-1835 & $2203-188$ & Q & 0.620 & 4.12 & 0.02 & 2.67 & (3.0) & -0.49 & $\mathrm{e}$ \\
\hline J2341-5816 & $2338-585$ & $\mathrm{EF}$ & $\ldots$ & 0.54 & $\ldots$ & $\ldots$ & & -1.18 & e \\
\hline
\end{tabular}

below $350 \mathrm{MHz}$ and so we classify it here as a CSS source. Slee (1995) gives 80 and $160 \mathrm{MHz}$ flux densities of $5 \mathrm{Jy}$ and $3.0 \mathrm{Jy}$ respectively. The snapshot VLBI observation of Fomalont et al. (2000) yielded a core-jet morphology, with the jet extending over $\sim 30$ mas form the core.

J1218-4600: the $80 \mathrm{MHz}$ flux density of Slee (1995) is $16 \mathrm{Jy}$.

J1242-0446 (3C275): dropped from the ATCA monitoring after the first few epochs and so no variability index or polarization information is given in Tingay et al. (2003b). VLA observations at 8.4 and $15 \mathrm{GHz}$ did not reveal any nuclear component (Mantovani et al. 1997) and, as suggested by that result, the source was not detected in the $4.8 \mathrm{GHz}$ VLBA observations of Fomalont et al. (2000).

J1546+0026: emission is seen on both sides of the core in the images of Stanghellini et al. (1999) and Fomalont et al. (2000), and also Peck \& Taylor (2000), who classify the source as a CSO.

J1556-7914: the observations of Lovell (1997) confirm the compact nature of the source on ATCA baselines, with $99 \%$ of the source flux density contained within an unresolved core component at $4.8 \mathrm{GHz}$.

\section{Newly identified CSS sources and candidates}

The twelve CSS sources and candidates found in the ATCA monitoring observations are listed in Table 4 and their spectra are plotted in Fig. 4. For selection, spectra were required to have a spectral index $\left(S \propto v^{+\alpha}\right)$ between 4.8 and $8.4 \mathrm{GHz}$ of less than $\sim-0.5$ and a peak, if any, below $\sim 500 \mathrm{MHz}$, and again we were also guided by the variability index and fractional polarization. Two sources have spectra indices marginally above the nominal cut-off of, e.g., Fanti et al. (1990), however we include them as candidates worthy of further study, as the nominal cut-off is purely a convention and does not have any particular physical meaning.

The variability indices are consistent with the low values observed in the previously reported CSS sources. Higher resolution observations are required to determine whether a number of these candidates satisfy the compactness requirement for CSS sources.

\subsection{Notes on individual sources}

J0059+0006 has a relatively high fractional polarization: only four sources have $p_{4.8}>7 \%$. The $4.8 \mathrm{GHz}$ VLBA observation of Fomalont et al. (2000) revealed a compact core with jet emission extending beyond 20 mas from the core.

J0119+0829: the snapshot VLBA images shows a curved jet extending over to the south with a bright component 30 mas north of the core (Fomalont et al. 2000).

J0155-4048: Lovell (1997) confirms the compact nature of the source on $6 \mathrm{~km}$ baselines, finding that $100 \%$ of the source flux density is contained within an unresolved core component at 4.8 GHz. The source is mostly resolved out on the shortest VLBA baselines (Fomalont et al. 2000). Emission appears on both sides of the core, suggestive of a CSO morphology, with the source extending over $\sim 20$ mas.

J0616-3456 was resolved with the VLA to a $2^{\prime \prime}$ source at $5 \mathrm{GHz}$ (Ulvestad et al. 1981) and was not detected in the VLBA observations of Fomalont et al. (2000).

J1351-1449: a borderline GPS/CSS source, with the data hinting at a spectral peak around $500 \mathrm{MHz}$. A core dominated source in the VLBA observations of Fomalont et al. (2000), with diffuse emission centred 20 mas north of the core.

J1424-4913: Lovell (1997) found that 95\% of the source flux density is contained within an unresolved core component on ATCA $6 \mathrm{~km}$ baselines at $4.8 \mathrm{GHz}$.

J1501-3918 has a strong core with possible short jet and a second component $\sim 130$ mas from the core in the VLBA image of Fomalont et al. (2000).

J1526-1351 (PMN J1526-1350): the source was mostly resolved out on $25 \mathrm{M} \lambda$ baselines at $4.8 \mathrm{GHz}$ (Fomalont et al. 2000). 

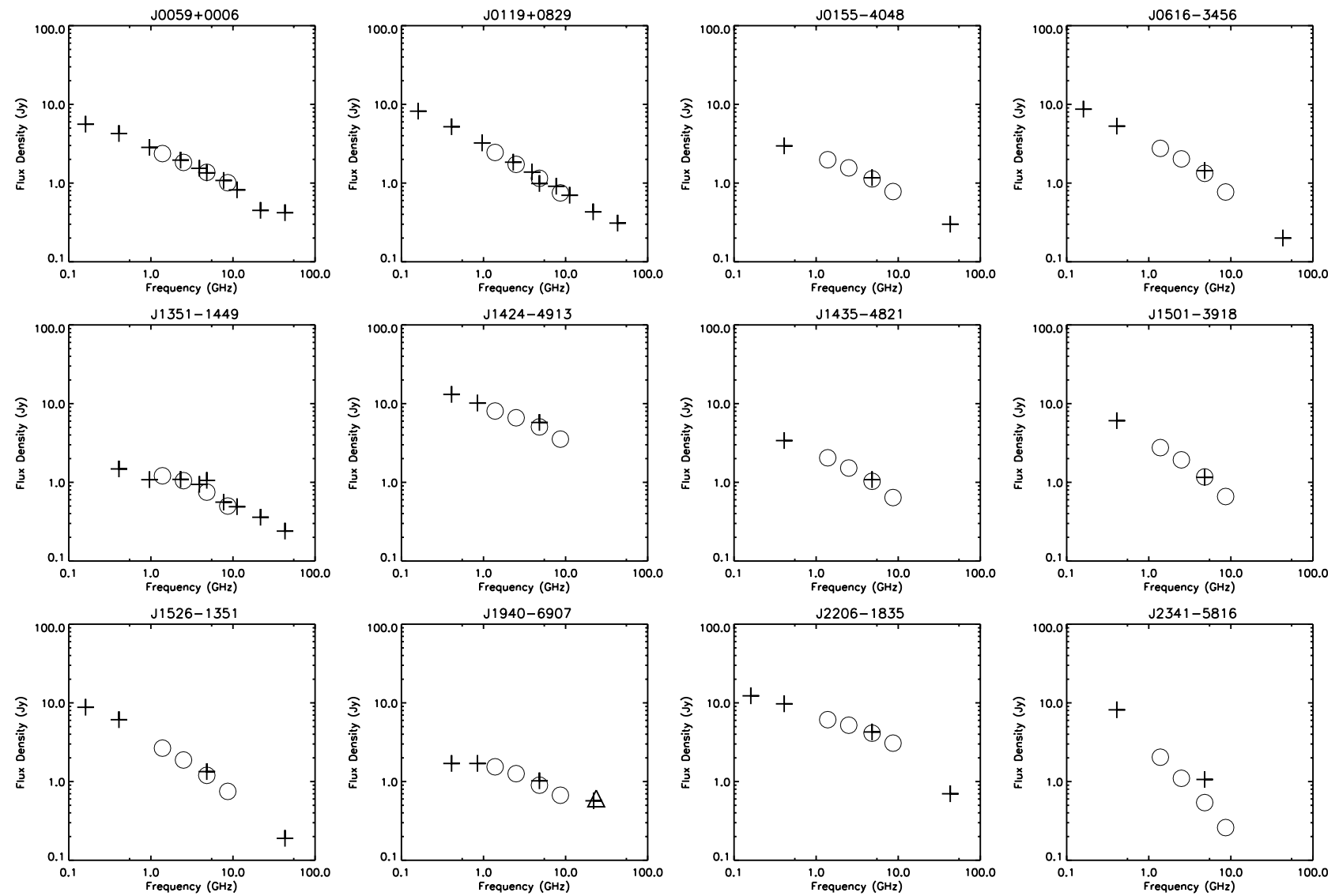

Fig. 4. Spectra of newly identified CSS sources. Symbols are the same as for Fig. 1.

J1940-6907 (PMN J1940-6908): the highest frequency points suggest a flattening of the spectrum at high frequencies. This would result in the source being rejected as a CSS candidate based on the strictest selection criteria, but may indicate the emerging visibility of a flat or inverted spectrum core. VLBI observations are required to determine the parsec-scale morphology.

J2206-1835: the source was resolved out on $10 \mathrm{M} \lambda$ baselines at $4.8 \mathrm{GHz}$ (Fomalont et al. 2000).

J2341-5816: dropped from the ATCA monitoring after the first few epochs so no variability index or fractional polarization is given by Tingay et al. (2003b).

\section{Other sources of interest}

A number of other sources have been suggested as GPS or CSS candidates in the literature. However, as discussed in this section, they do not meet one of more of the accepted criteria for GPS or CSS sources. The ATCA monitoring program included 7 of the 12 "new sources with GPS-type spectra" and 7 of the 8 "other sources with inverted spectra" given by Tornikoski et al. (2001). As noted by Tornikoski et al. (2001), many of these sources show evidence for significant variability, and this is reflected by the fact that 11 of the 14 sources had variability indices at $4.8 \mathrm{GHz}$ (derived from the ATCA monitoring program) above the median value. For many of these sources the spectra based on the average fluxes over the epochs of the ATCA monitoring did not meet our criteria for GPS or CSS sources. Tornikoski et al. (2001) point out that many of the sources they consider have higher spectral peaks than most catalogued GPS sources, and so some of these may be related to the class of High Frequency Peakers (HFPs), which are somewhat more variable than GPS/CSS sources (Dallacasa et al. 2000, 2002; Dallacasa 2003). Table 5 and Fig. 5 contain a number of the sources from Tornikoski et al. (2001), and in addition a number of sources from our ATCA monitoring with inverted spectra up to $8.4 \mathrm{GHz}$ - candidate HFPs. We also include J1419-1928, designated as a candidate CSO by Taylor \& Peck (2003).

It is notable that the sources here generally have higher variability indices and fractional polarizations than those in the preceding tables. In addition, larger differences are evident in the fractional polarizations measured by Perley (1982) and those measured in the ATCA monitoring. The tendency for increased variability in sources with more inverted spectra (e.g., Kesteven et al. 1977; Tingay et al. 2003b) is seen in the variability indices: in the sample of 185 sources only $8(4.3 \%)$ have variability indices at $4.8 \mathrm{GHz}$ of 0.30 or higher, with three of these appearing in Table 5.

\subsection{Notes on individual sources}

J0106-4034 has the fourth highest 4.8 to $8.4 \mathrm{GHz}$ spectral index in the ATCA sample. PKSCAT flux densities of $0.57 \mathrm{Jy}$ at 
Table 5. Other sources of interest. Sources for which no reference is given are those from the ATCA program with spectra that are inverted, but which do not meet our criteria for inclusion as GPS candidates. The spectral index, $\alpha$, is that determined between 4.8 and $8.4 \mathrm{GHz}$ from the ATCA monitoring (Tingay et al. 2003b). Parameters are the same as for previous tables.

\begin{tabular}{|c|c|c|c|c|c|c|c|c|c|c|}
\hline Source & B1950 & Refs. $^{a}$ & Type & $z$ & $\begin{array}{l}S_{4.8} \\
(\mathrm{Jy})\end{array}$ & $m_{4.8}$ & \multicolumn{2}{|c|}{$\begin{array}{l}p_{4.8} \\
(\%)\end{array}$} & $\alpha$ & Flag \\
\hline J0106-4034 & 0104-408 & & $\mathrm{Q}$ & 0.584 & 3.56 & 0.06 & 1.34 & (1.4) & 0.57 & $\mathrm{c}$ \\
\hline J0457-2324 & $0454-234$ & T01 & Q & 1.003 & 2.04 & 0.15 & 1.44 & (3.5) & 0.23 & 1 \\
\hline J0538-4405 & $0537-441$ & T01 & BL & 0.894 & 3.48 & 0.30 & 1.28 & (1.0) & 0.15 & 1 \\
\hline J1035-2011 & $1032-199$ & & Q & 2.198 & 1.01 & 0.11 & 3.03 & (4.1) & 0.52 & 1 \\
\hline $\mathrm{J} 1058-8003$ & $1057-797$ & T01 & $\mathrm{Q}$ & $\ldots$ & 2.03 & 0.20 & 3.14 & & 0.16 & c \\
\hline J1337-1257 & $1334-127$ & T01 & Q & 0.539 & 3.55 & 0.24 & 3.34 & & 0.41 & 1 \\
\hline J1419-1928 & $1417-192$ & T03 & $\mathrm{G}$ & 0.120 & 0.47 & 0.07 & 2.44 & & -0.03 & $\mathrm{e}$ \\
\hline $\mathrm{J} 1743-0350$ & $1741-038$ & & Q & 1.054 & 4.43 & 0.11 & 0.96 & (1.2) & 0.29 & $\mathrm{c}$ \\
\hline $\mathrm{J} 1751+0939$ & $1749+096$ & & $\mathrm{BL}$ & 0.322 & 2.41 & 0.28 & 1.41 & (6.1) & 0.61 & c \\
\hline J1957-3845 & $1954-388$ & T01 & $\mathrm{Q}$ & 0.626 & 3.21 & 0.26 & 3.09 & (1.0) & 0.05 & $\mathrm{c}$ \\
\hline $\mathrm{J} 2123+0535$ & $2121+053$ & & Q & 1.878 & 0.95 & 0.32 & 1.17 & (2.5) & 0.54 & $\mathrm{c}$ \\
\hline J2131-1207 & $2128-123$ & T01 & Q & 0.499 & 2.55 & 0.04 & 1.20 & (0.6) & 0.09 & $\mathrm{c}$ \\
\hline $\mathrm{J} 2148+0657$ & $2145+067$ & T01 & $\mathrm{Q}$ & 0.990 & 6.33 & 0.05 & $<0.5$ & (1.0) & 0.25 & $\mathrm{c}$ \\
\hline J2239-5701 & $2236-572$ & & $\mathrm{EF}$ & $\ldots$ & 0.72 & 0.05 & 4.39 & & 0.24 & $\mathrm{c}$ \\
\hline J2258-2758 & $2255-282$ & T01 & Q & 0.926 & 3.37 & 0.10 & 0.92 & & 0.89 & 1 \\
\hline $\mathrm{J} 2358-1020$ & $2355-106$ & & $\mathrm{Q}$ & 1.626 & 1.11 & 0.34 & 2.84 & & -0.18 & $\mathrm{c}$ \\
\hline
\end{tabular}

${ }^{a}$ References: T01 = Tornikoski et al. (2001); T03 = Taylor \& Peck (2003).

$2.7 \mathrm{GHz}$ and $0.85 \mathrm{Jy}$ at $5.0 \mathrm{GHz}$ differ significantly from corresponding values of 1.64 and $3.56 \mathrm{Jy}$ from the ATCA monitoring. The PMN $4.85 \mathrm{GHz}$ flux density of $2584 \pm 99 \mathrm{mJy}$ (Wright et al. 1994) and high frequency flux densities evident in Fig. 5 also suggest significant variability, although the variability index is quite moderate. Kedziora-Chudczer et al. (2001) report evidence for intra-day variability at $2.4 \mathrm{GHz}$.

J0457-2324: noted as a new source with a GPS-type spectrum by Tornikoski et al. (2001), with turnover at $3 \mathrm{GHz}$. Possible core-jet morphology in the VLBA observations of Fomalont et al. (2000).

J0538-4405: noted as a new source with a GPS-type spectrum by Tornikoski et al. (2001), with spectral peak at $5 \mathrm{GHz}$.

J1035-2011: a turn-up at low frequencies is apparent. The 4.8 GHz image of Fomalont et al. (2000) shows a core-jet morphology.

J1058-8003: identified as a quasar by Jauncey et al. (1989). Noted as a new source with a GPS-type spectrum by Tornikoski et al. (2001), with a peak at $10 \mathrm{GHz}$, and the spectrum in Fig. 5 is consistent with this. Beasley et al. (1997) measured a flux density of $0.41 \mathrm{Jy}$ at $147 \mathrm{GHz}$, indicating the spectrum steepens further at high frequencies. The turn-up at low frequencies notwithstanding, the spectrum of the relatively sparsely observed source is consistent with that of GPS sources. However, as the variability indices at all four frequencies of the ATCA monitoring were relatively high we do not include it as a candidate GPS source, although it is worthy of further study.

J1337-1257: noted as a new source with a GPS-type spectrum by Tornikoski et al. (2001), with turnover at $20 \mathrm{GHz}$. The ATCA data have a spectral index of $\sim 0.4$, though the data of
Kovalev et al. (1999) suggest the spectrum is not as inverted as this. Very compact in the VLBA observations of Fomalont et al. (2000).

J1419-1928: this galaxy has been noted as a candidate CSO by Taylor \& Peck (2003) based on their 5 and $15 \mathrm{GHz}$ imaging. As noted by Fassnacht \& Taylor (2001), while there is some overlap between CSOs (defined by their morphology) and GPS/CSS sources (defined largely by their spectra) there is no clear correspondence between the two classes. Certainly the spectrum based on the ATCA data in Fig. 5 would not invite classification as either a GPS or CSS source. The higher PMN flux density of $1.021 \mathrm{Jy}$ at $4.85 \mathrm{GHz}$ (Griffith et al. 1994), together with the "e" structure flag, suggest the source is partially resolved out on the $6 \mathrm{~km}$ ATCA baseline. Excluding the ATCA points yields a CSS-like spectrum with a spectral index of -0.6 .

J1743-0350: the variability index at $4.8 \mathrm{GHz}$ is moderate, however the ATCA $4.8 \mathrm{GHz}$ flux density of $4.43 \mathrm{Jy}$ is significantly greater than the 2.37 Jy of the PMN (Griffith et al. 1995). VSOP space VLBI observations are described by Wajima et al. (2000).

J1751+0939: this source has the third most inverted spectrum of the ATCA sample of 202 sources, with a 4.8 to $8.4 \mathrm{GHz}$ spectral index of 0.61 . The fractional polarization of $6.1 \%$ measured by Perley (1982) is much higher than the ATCA value of $1.41 \%$. There is a pronounced turn-up at low frequencies.

J1957-3845: noted as a new source with a GPS-type spectrum by Tornikoski et al. (2001), with turnover at $\sim 10 \mathrm{GHz}$. The compilation of data in Fig. 5 would suggest that the spectral peak lies closer to $8 \mathrm{GHz}$. A compact core-jet morphology was revealed by the VLBA observations of Fomalont et al. (2000). 

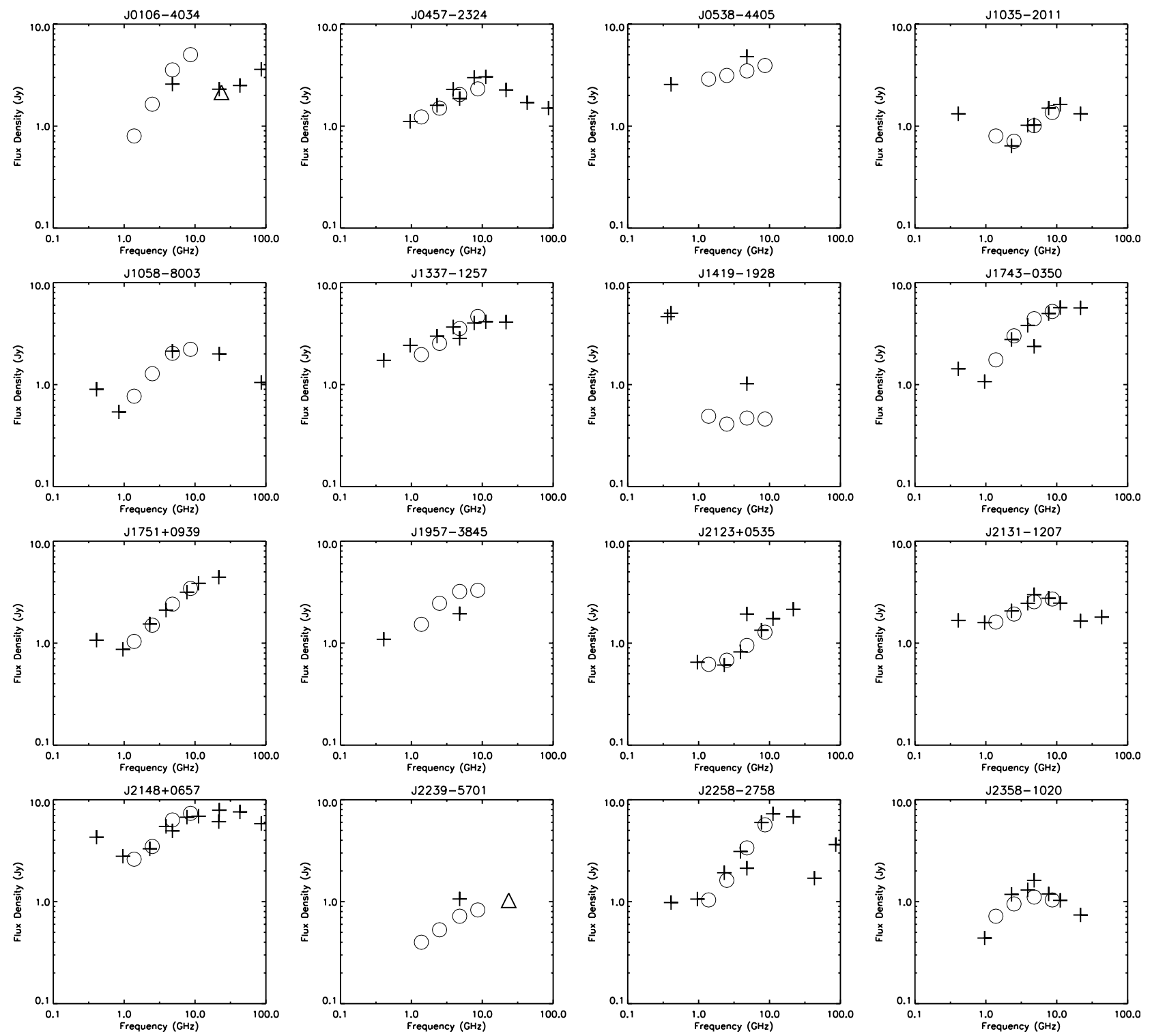

Fig. 5. Spectra of other sources of interest. Symbols are the same as for Fig. 1.

J2123+0535: the spectral index between 4.8 and $8.43 \mathrm{GHz}, 0.54$, is the fifth highest of the ATCA sample. In addition, the PMN flux density of $1.93 \mathrm{Jy}$ (Griffith et al. 1995 ) is almost double the ATCA value. The VLBA observations of Fomalont et al. (2000) revealed a bright core and a compact secondary component 1.2 mas away.

J2131-1207: noted as a new source with a GPS-type spectrum by Tornikoski et al. (2001), with turnover at $5 \mathrm{GHz}$. The $80 \mathrm{MHz}$ flux density of 6 Jy (Slee 1995) indicates a large turnup at low frequencies. Compact core-jet morphology in the VLBA observations of Fomalont et al. (2000).

J2148+0657: noted as a source with an inverted spectrum by Tornikoski et al. (2001). The compilation of data in Fig. 5 reveals a turn-up at low frequencies and a peak around $30 \mathrm{GHz}$. The variability index is quite low, however, as noted by Tornikoski et al. (2001), over longer timescales the source is strongly variable. The fractional polarization upper limit is consistent with those of GPS/CSS sources. A compact core-jet morphology was seen in the VLBA observations of Fomalont et al. (2000).

J2239-5701: a relatively poorly studied source with a low variability index in the ATCA monitoring. The ATCA $4.8 \mathrm{GHz}$ flux density lies between the PKSCAT value of 0.49 Jy (Wright \& Otrupcek 1990) and the PMN value of $1.063 \pm 0.056$ (Wright et al. 1994), indicating moderate longer term variability. Our $22 \mathrm{GHz}$ measurement in April 2003 suggests the spectral peak lies (for that epoch, at least) above $20 \mathrm{GHz}$.

J2258-2758: noted as a new source with a GPS-type spectrum by Tornikoski et al. (2001), with a peak at $20 \mathrm{GHz}$. The 4.8 to $8.4 \mathrm{GHz}$ spectral index was the highest of the ATCA sources. The variability index is only just above the median, however the spectra compiled by Tornikoski et al. (2001) reveals variations of almost an order of magnitude in flux densities at $\mathrm{mm}$ 
wavelengths. A compact core-jet morphology was seen in the VLBA observations of Fomalont et al. (2000).

J2358-1020 has the most GPS-like spectrum of the sources in Fig. 5. The spectral index steepens from -0.18 between 4.8 and $8.6 \mathrm{GHz}$ (ATCA data) to -0.50 between 11.2 and $21.65 \mathrm{GHz}$ (Kovalev et al. 1999), consistent with the requirements for a GPS source. However, as the source has one of the largest variability indices at 4.8 and $8.6 \mathrm{GHz}$ in the ATCA monitoring, we do not include it as a candidate. Variability is also apparent on longer timescales, as the ATCA $4.8 \mathrm{GHz}$ flux density is intermediate between the $0.63 \mathrm{Jy}$ PKSCAT value (Wright $\&$ Otrupcek 1990) and the $1.618 \pm 0.085$ Jy of PMN (Griffith et al. 1994). Furthermore, inspection of Fig. 2 of Tingay et al. (2003b) indicates that the spectrum changes significantly over time, with spectral indices of $\sim 0.0$ all the way from 1.4 to 8.6 GHz at some epochs. The $4.8 \mathrm{GHz}$ VLBA observations of Fomalont et al. (2000) yielded a very compact, core-dominated source.

\section{Discussion}

\subsection{Incidence of GPS and CSS sources}

There are 28 GPS sources and candidates in Tables 1 and 2, constituting $14 \%$ of the sample of 202 sources, in general agreement with the $\sim 10 \%$ incidence rate given by O'Dea (1998). Tables 3 and 4 contain 20 CSS sources, $10 \%$ of the sample. This is, as expected, significantly lower than the $\sim 30 \%$ given by O'Dea (1998), as there was a bias toward flatspectrum sources in the compilation of the 202 sources.

\subsection{Spectral width}

In the sample of 15 sources of O'Dea et al. (1991), 0108+388 had the narrowest spectrum with a $F W H M$ of $\sim 0.95$. O'Dea et al. note that a width of 0.77 decades of frequency is close to the minimum for an ideal source - a homogeneous selfabsorbed synchrotron source with a power law electron energy spectrum. The presence of multiple components, or of inhomogeneities, will naturally broaden the width. As noted in Sect. 2.1, J1939-6342 (1934-638) has the narrowest width of the previously identified GPS sources, with a value similar to that of $0108+388$. J1939-6342 has a compact double (CD) morphology, and although $0108+388$ was initially considered a CD, it is now recognised as a CSO (Conway et al. 1994; Taylor et al. 1996). Comparison with studies of other GPS sources in the literature reveals that this appears to be a characteristic of $\mathrm{CD} / \mathrm{CSO}$ sources. For example, the other two CD sources in the sample of 15 GPS sources of O'Dea et al. (1991), 0019-000 and 1225+368, have spectral FWHMs of $1 \sim 1.25$ decades of frequency, at the low end of the distribution.

To quantify this further, we have examined the sample of fainter GPS sources of Snellen et al. (2000), which is presented with spectra compiled from total flux density measurements and morphologies determined from VLBI observations. (The criteria for morphological classification are given in Snellen et al. (2000): we note here that the CD classification required

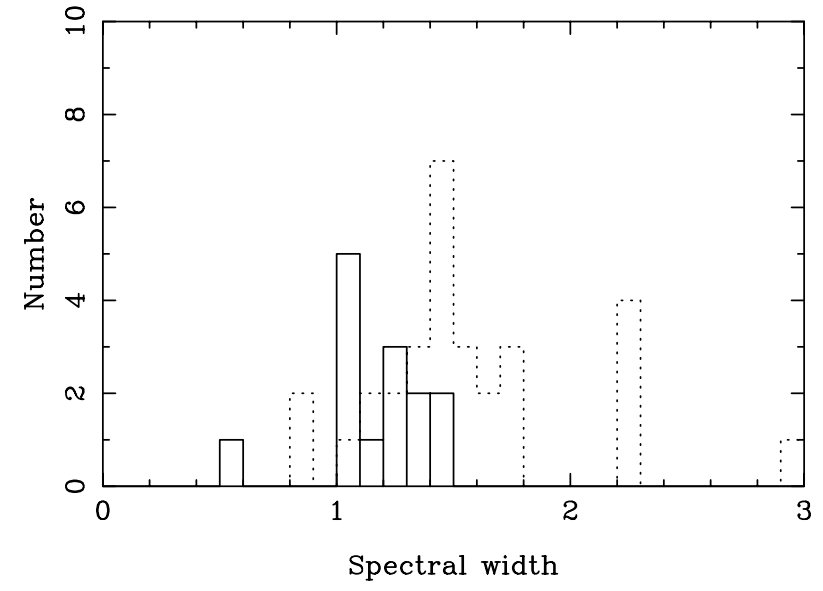

Fig. 6. Spectral width ( $F W H M$ in decades of frequency) for the $\mathrm{CD} / \mathrm{CSO}$ sources (solid line histogram) and other sources (dotted line histogram) from the sample of 44 sources of Snellen et al. (2000).

similar spectra in both components and not necessarily similar flux densities.) In Fig. 6, the distributions of spectral widths are plotted for the $14 \mathrm{CD} / \mathrm{CSO}$ sources and the 30 other sources from Snellen et al. (2000). A Kolmogorov-Smirnov (KS) test comparing two distributions yields a probability that the two are drawn from the same distribution of $0.6 \%$. A more conservative estimate, obtained by excluding the narrowest $\mathrm{CD} / \mathrm{CSO}$ width and the largest "other morphologies" width, is $1.4 \%$. Such a trend is plausible, as $\mathrm{CD} / \mathrm{CSO}$ sources are believed to lie close to the plane of the sky and/or have generally small advance speeds, resulting in emission on both sides of the core being visible. In contrast, core-jet sources have spectra in which a flatter spectrum core component makes an appreciable contribution, resulting in a broader peak. In addition to the intrinsically narrower spectra, it may be that $\mathrm{CD}$ and CSO sources may also have a sharper low frequency cut-offs due to increased free-free absorption and/or synchrotron selfabsorption. There is, however, appreciable overlap between the two distributions, and so the spectral width does not have a great predictive power in individual cases as to the morphology. It will, however, be of interest to determine the morphology of J1726-6427, the newly identified GPS with a narrow spectrum (Fig. 2).

We have also compared the distribution of widths of all 28 sources in Tables 1 and 2 against all 44 sources in the Snellen et al. (2000) sample: the two distributions have a probability of $88 \%$ of being drawn from the same distribution.

\subsection{Redshift distribution}

As mentioned in Sect. 1, a significant fraction of sources with $\mathrm{GHz}$ peaked spectra have been found to lie at high-redshift. This is borne out by the distribution of redshifts in Table 1. We can compare this with, e.g., the distribution of redshifts for 277 sources in flat-spectrum sample of Drinkwater et al. (1997). In that sample, $45 \%$ of sources had $z<1,38 \%$ had $1<z<2$, $13 \%$ had $2<z<3$ and $4 \%$ had $z>3$. In contrast we find that 8 of the 17 (47\%) GPS sources with known 


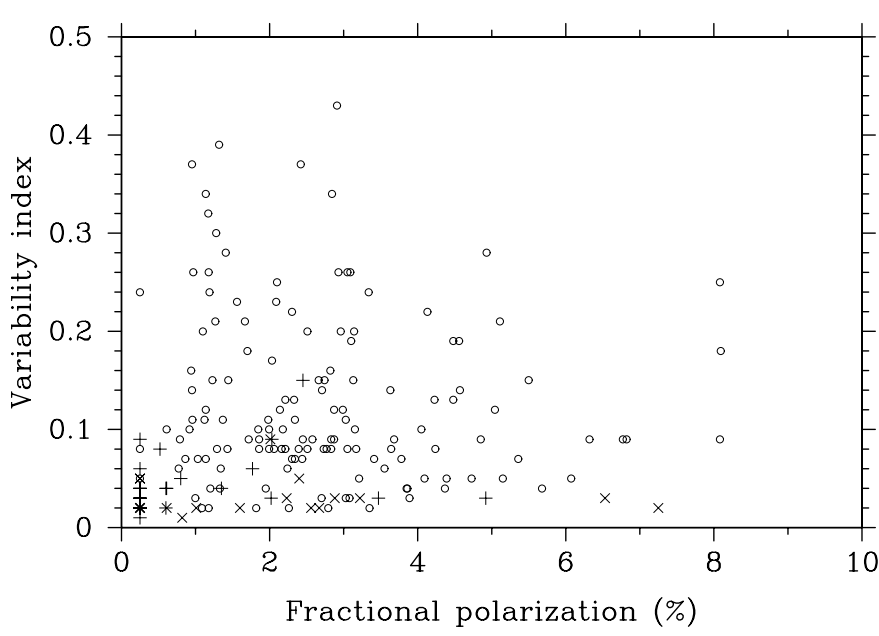

Fig. 7. Variability index as a function of percentage linear polarization, for the $4.8 \mathrm{GHz}$ ATCA monitoring data (Tingay et al. 2003b). Plus symbols are for the 28 GPS sources in Tables 1 and 2, crosses for the 18 CSS sources in Tables 3 and 4 for which these parameters were measured, and open circles for the 139 other sources. All the points with a plotted fractional polarization of 0.25 represent upper limits of 0.5. A number of data points are over-plotted, particularly for GPS and CSS sources at low variability index and low polarization: there are, for example, 7 GPS sources with an $m_{4.8}$ of 0.02 and $p_{4.8}<0.5$.

redshifts in Table 1 have $z>2$, and 4 of 17 (24\%) have $z>3$. Of the newly identified GPS sources in Table 2, two of five $(40 \%)$ have $z>2$. The samples are small but the expected trend is indeed observed.

The known and newly identified CSS sources and candidates show the opposite trend: five of seven sources $(71 \%)$ in Table 3 and five of seven $(71 \%)$ in Table 4 have $z<1$. The marked difference from the distribution of Drinkwater et al. is not unexpected, as once again the CSS sources have steep spectra whereas the Drinkwater et al. sample was for flat-spectrum sources. Of the 42 sources with known redshifts in the sample of CSS sources of Fanti et al. (1990), 64\% had $z<1$ and $29 \%$ had $1<z<2$, in broad agreement with the distributions for the sample here.

In contrast, the distribution of redshifts for the sources in Table 5 (neglecting the candidate CSO J1419-1928) has 8 of $13(61 \%)$ sources with $z<1$, four $(31 \%)$ with $1<z<2$, and one $(8 \%)$ with $z>2-$ much closer to the distribution of flat-spectrum quasars of Drinkwater et al. (1997). (We note that there is some overlap between these samples, though the effect of this is not significant.)

\subsection{Variability}

Comparing the $4.8 \mathrm{GHz}$ variability indices in Tables $1-4$ with the overall range and the median value $(0.08)$ given in Sect. 1 reveals that both the previously reported and newly identified GPS and CSS sources have low variability indices. This can also be seen in Fig. 7, where the $4.8 \mathrm{GHz}$ variability index has been plotted against fractional polarization, for all 185 sources monitored in the ATCA program. J1522-2730, previously reported as a GPS source, has a relatively high variability index and may, as noted by Tornikoski et al. (2001) only show a GPS-like spectrum at some epochs.

More quantitatively, we can compare the distributions of variability indices for the three classes plotted in Fig. 7. The $\mathrm{KS}$ test confirms that the probability that the distribution of the 28 GPS (previously reported and new candidate) sources is drawn from the same distribution as for the 139 other (i.e., neither GPS nor CSS) sources is $<10^{-6}$. The comparison of CSS (previously reported and new candidate) sources and other sources yields a similar result. The probability that the distributions of GPS and CSS sources are drawn from the same distribution is, in contrast, $19 \%$.

Aller et al. (2002) have studied the long term variability of a sample of 32 GPS sources and find that several sources exhibit variability comparable to those seen in flat-spectrum, coredominated radio sources. Ten of 18 sources with good longterm monitoring data show variability at some level, though the timescales are typically longer than for flat-spectrum, coredominated radio sources. This is also seen for a number of sources in this paper, which have low variability indices over the 3.5 year period of the ATCA monitoring, but which have clearly varied on longer timescales when comparing the mean ATCA flux density with those from the PMN and PKS catalogs.

Tornikoski et al. (2001) note, and we confirm, that the majority of their sources with GPS-type spectra show higher variability than the established GPS sources. GPS-like spectra may be observed during certain stages of large radio outbursts in flat-spectrum AGN, however the inverted ATCA spectra in Fig. 5 appear, in comparison to the admittedly small amount of supplementary data, to be relatively persistent. A number have higher turnover frequencies and/or quite inverted spectra, and so these may be related to High Frequency Peakers (Dallacasa 2003).

\subsection{Fractional polarization}

As was done for the variability indices, we can compare the distributions of fractional polarization at $4.8 \mathrm{GHz}$ for the three classes plotted in Fig. 7. The probability that the distribution of the GPS sources is drawn from the same distribution as for the "other" sources is $<10^{-6}$. For CSS sources, the probability is $3 \%$. This is influenced by two CSS sources - the previously reported J0627-0553, and new candidate J0059+0006 - with fractional polarizations $>6 \%$. The probability that the distributions of GPS and CSS sources are drawn from the same distribution is $3 \%$. These results are in line with previous reports that CSS sources tend to have higher fractional polarizations than GPS sources (see, e.g., O’Dea 1998).

Nevertheless, sources with low variability indices and low fractional polarizations are much more likely to be GPS or CSS sources. Of the 24 sources with fractional polarizations below $0.5 \%, 21$ appear in Tables 1 to 4 . Of the remaining three, one is listed in Table 5, and the other two are the extended radio galaxy Cen A and the gravitational lens system J1833-2103, both of which are complex sources not well-characterised by the snapshot observing mode used for the ATCA monitoring program (Tingay et al. 2003b). 

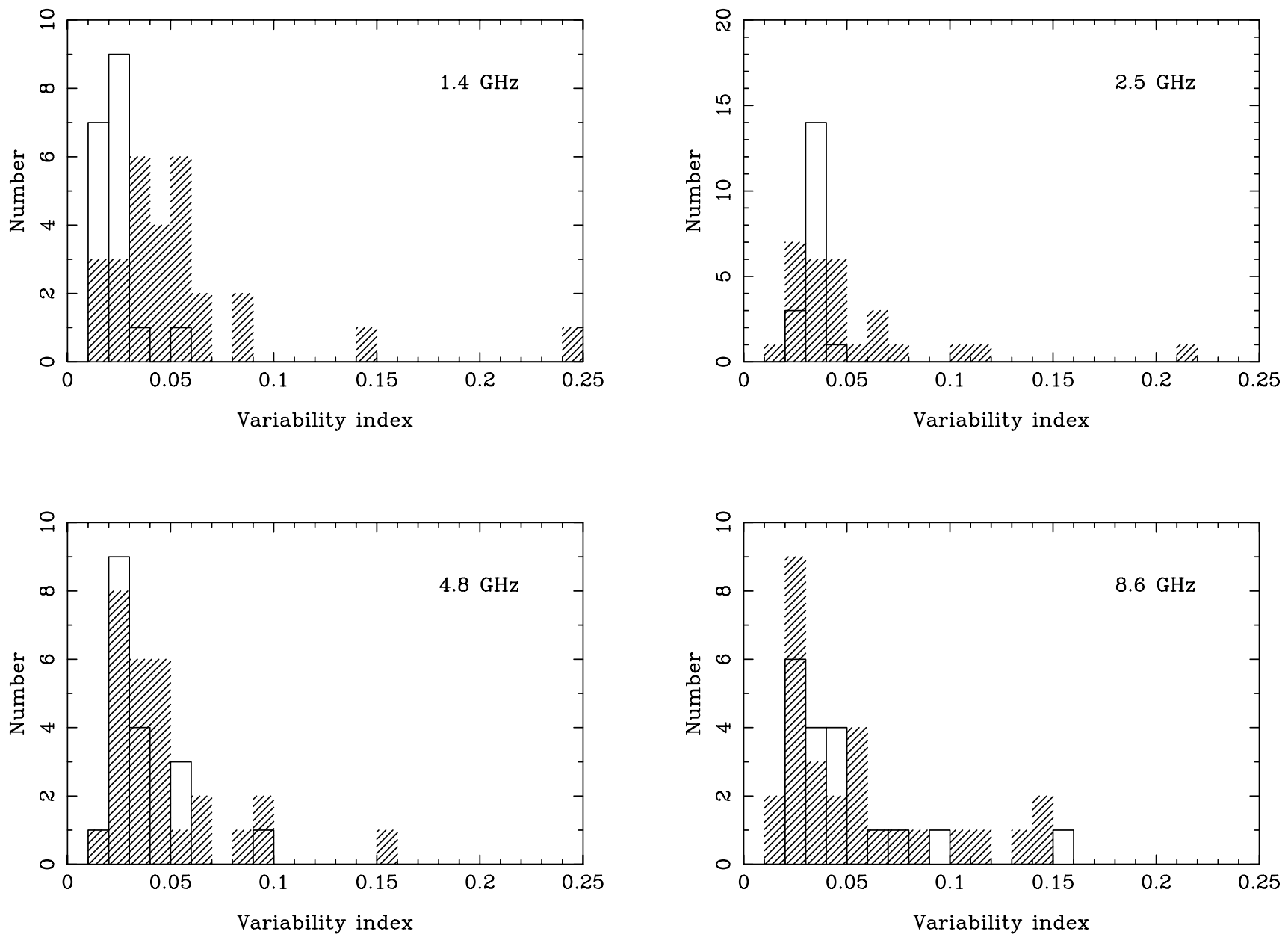

Fig. 8. Distributions of variability index for GPS (hatched) and CSS (solid outline) sources. The distributions differ significantly at 1.4 and 2.5 GHz, with CSS sources tending to be less variable.

\subsection{Behaviour at other frequencies}

For the reasons outlined in Sect. 1, we have focused on the characteristics of sources at $4.8 \mathrm{GHz}$. However, as the ATCA monitoring program was undertaken at four frequencies, we are able to check whether the conclusions drawn at $4.8 \mathrm{GHz}$ also apply at other centimeter frequencies. We have performed the same KS tests as those described in the previous two sections for variability and fractional polarization distributions at 1.4, 2.5 and $8.6 \mathrm{GHz}$.

At all frequencies, the distributions of variability indices of both GPS and CSS sources differ significantly (probabilities of being drawn from the same distribution of $<0.1 \%$ ) from those of the "other" sources. At $1.4 \mathrm{GHz}$ the distributions of GPS and CSS sources are significantly different, with the CSS sources being on average less variable than the GPS sources. At $2.5 \mathrm{GHz}$ the probability they are drawn from the same distribution is $1 \%$, which increases to $19 \%$ at $4.8 \mathrm{GHz}$ and $69 \%$ at $8.6 \mathrm{GHz}$. As shown in Fig. 8, at 1.4 and $2.5 \mathrm{GHz}$, the distributions of CSS sources are concentrated at lower variability indices. The most variable GPS source at 1.4, 2.5 and $4.8 \mathrm{GHz}$ is $\mathrm{J} 1522-2730$ which, as discussed in Sect. 2.1, is arguably not a bona fide member of this class, however the significant differences in distributions remain even if this source is excluded. The lower variability of CSS sources at low frequencies is also consistent with the more general trend (mentioned in Sect. 6) for increased variability as the spectral index increases.

The distributions of fractional polarization of GPS sources differ significantly at all frequencies from those of "other" sources. However, as frequency increases, the fractional polarization distributions of CSS sources become more similar to those of "other" sources, from being significantly different at 1.4 and $2.5 \mathrm{GHz}$, to a probability of $3 \%$ at $4.8 \mathrm{GHz}$, and a probability of $31 \%$ at $8.6 \mathrm{GHz}$. The tendency for CSS to become more polarized with increasing frequency (at least at centimeter wavelengths) has been noted previously (see, e.g., O'Dea 1998). As a result, the distributions of GPS and CSS sources tend to become less similar with increasing frequency: the $\mathrm{KS}$ probabilities are $51 \%$ at $1.4 \mathrm{GHz}, 73 \%$ at $2.5 \mathrm{GHz}, 3 \%$ at $4.8 \mathrm{GHz}$ and $5 \%$ at $8.6 \mathrm{GHz}$.

It should be kept in mind that, although the new candidate GPS and CSS sources have been selected primarily based on their spectra, the variability indices and fractional polarizations have also been considered. Thus the probabilities given above will, to some degree, reflect this. The general trends that are observed, however, are unlikely to be significantly affected. 


\subsection{Low frequency turn-ups}

Early searches for GPS sources rejected candidates with a turn-up at low frequencies (e.g., Gopal-Krishna et al. 1983). However, given the evidence that at least some GPS sources are undergoing renewed activity, it is possible that some GPS sources will have the GPS spectrum from the central kpcscale region imposed upon the extended steep spectrum component, resulting in an overall spectrum that turns up at low frequencies. In Fig. 1 it is apparent that J0241-0815 (NGC1052), $\mathbf{J} 1522-2730$, and $\mathbf{J} 2136+0041$ show departures from the fitted spectral form. Similar behaviour is seen for the new candidates J1624-6809 and, possibly, J1837-7108 (Fig. 2). In extreme cases, such as J0745-0044, J0900-2808 (both Fig. 1) and J1658-0739 (Fig. 2), the spectra clearly turn up at low frequencies. However, these sources have clear spectral peaks at $\mathrm{GHz}$ frequencies, and display the variability and polarization properties of other GPS sources. Imaging observations are required to determine whether the low frequency component corresponds to extended emission within which the $\mathrm{GHz}$-peaked spectrum component is embedded.

\subsection{Compactness}

In the ATCA monitoring program, as described in Sect. 1, a flag was assigned according to the degree of compactness of a source on the maximum $6 \mathrm{~km}$ baseline (see also Tingay et al. 2003b). GPS sources are expected to be confined to sub-kpc scales, with CSS sources generally $<20 \mathrm{kpc}$ in extent. This trend is seen in the distribution of flags for Tables 1 and 2 (18 "c", 3 "e", 7 "l") as compared to those of Table 3 and 4 (9 "c", 9 "e", 3 "l").

We can investigate this more quantitatively by comparing the mean ATCA $4.8 \mathrm{GHz}$ correlated flux density on the $6 \mathrm{~km}$ baseline with the sum of the model-fit components from the $4.8 \mathrm{GHz}$ VLBA observations of June 1996 (Fomalont et al. 2000). The results are shown in Fig. 9. The expected trend is seen, with most of the flux density of GPS sources being recovered in the VLBI observations, whereas the CSS sources were generally partially (and in some cases completely) resolved.

\section{Conclusions}

The multi-frequency, multi-epoch monitoring of 185 radio sources (and multi-frequency data of 17 more) with the Australia Telescope Compact Array has provided a data set well-suited to searching for new GPS and CSS candidates. We have studied the properties of previously reported GPS and CSS sources, and identified eight new candidate GPS sources and 12 new candidate CSS sources with similar properties. VLBI studies are required for a number of these candidates to determine whether they satisfy the compactness requirements for membership of their respective classes. The incidence of GPS sources in the ATCA sample is in agreement with that of other samples, with the incidence of CSS sources being lower than that of other samples due to the preference given to flatter spectral index sources in selecting the ATCA sample. We have confirmed that GPS sources are generally

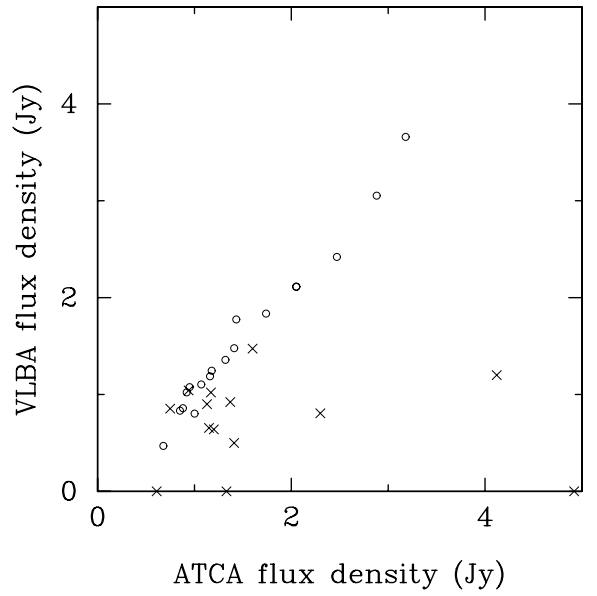

Fig. 9. Comparison of mean $4.8 \mathrm{GHz}$ flux density on the ATCA $6 \mathrm{~km}$ baseline (Tingay et al. 2003b) with the total model-fit flux density from the $4.8 \mathrm{GHz}$ VLBA pre-launch survey observations in June 1996 (Fomalont et al. 2000). GPS sources and candidates are plotted with circles, and CSS sources and candidates are plotted with crosses. For clarity, one GPS source has been excluded from this plot: $\mathrm{J} 2136+0041$, which has an ATCA flux density of $9.47 \mathrm{Jy}$ and a modelfit VLBA flux density of $10.13 \mathrm{Jy}$. The expected trend is observed, with GPS sources being more compact (i.e. having most of the flux density recovered on VLBI baselines) than CSS sources.

compact on $6 \mathrm{~km}$ baselines, and that in most cases the ATCA flux densities are in good agreement with the flux densities recovered in VLBI imaging, whereas CSS sources are in general less compact and are sometimes completely resolved in VLBI observations. The ATCA monitoring at $4.8 \mathrm{GHz}$ confirms the expectation that both GPS and CSS sources have low variability indices and low fractional polarizations. These conclusions hold true for the other frequencies, though with the fractional polarization of CSS sources seen to increase with frequency, as has been previously noted. We also find that CSS sources are on average less variable at 1.4 and $2.5 \mathrm{GHz}$ than GPS sources. Based on qualitative observations of sources in the ATCA sample, and a quantitative study of the faint GPS sample of Snellen et al. (2000), we have also found that GPS sources with Compact Double and Compact Symmetric Object morphologies tend to have narrower spectra than other classes of GPS sources, compatible with a higher degree of homogeneity in these sources, a larger viewing angle to the jet axis, and/or increased absorption at lower frequencies.

Acknowledgements. This research has made use of NASA's Astrophysics Data System Bibliographic Services, and the NASA/IPAC Extragalactic Database (NED) which is operated by the Jet Propulsion Laboratory, California Institute of Technology, under contract with the National Aeronautics and Space Administration. Ignas Snellen is thanked for helpful discussions, and the referee is acknowledged for comments and suggestions which have resulted in an improved paper.

\section{References}

Aller, M. F., Aller, H. D., Hughes, P. A., \& Plotkin, R. M. 2002, Proc. 6th European VLBI Network Symp., ed. E. Ros, R. W. Porcas, A. P. Lobanov, \& J. A. Zensus, 131 
Beasley, A. J., Conway, J. E., Booth, R. S., Nyman, L.-A., \& Holdaway, M. 1997, A\&AS, 124, 469

Bolton, J. G., Gardner, F. F., \& Mackey, M. B. 1963, Nature, 199, 682 Conway, J. E., Myers, S. T., Pearson, T. J., et al. 1994, ApJ, 425, 568 Conway, J. E. 2002, New Astron. Rev., 46, 263

Dallacasa, D., Stanghellini, C., Centonza, M., \& Fanti, R. 2000, A\&A, 363, 887

Dallacasa, D., Stanghellini, C., Centonza, M., \& Furnari, G. 2002, New Astron. Rev., 46, 299

Dallacasa, D. 2003, PASA, 20, 79

de Vries, W. H., Barthel, P. D., \& Hes, R. 1995, A\&AS, 114, 259

de Vries, W. H., Barthel, P. D., \& O’Dea, C. P. 1997, A\&A, 321, 105

de Vries, W. H., O'Dea, C. P., Barthel, P. D., \& Thompson, D. J. 2000, A\&AS, 143, 181

Douglas, J. N., Bash, F. N., Bozyan, F. A., Torrence, G. W., \& Wolfe, C. 1996, AJ, 111, 1945

Drinkwater, M. J., Webster, R. L., Francis, P. J., et al. 1997, MNRAS, 284, 85

Fanti, R., Fanti, C., Schilizzi, R. T., et al. 1990, A\&A, 231, 333

Fassnacht, C. D., \& Taylor, G. B. 2001, AJ, 122, 1661

Fey, A. L., Clegg, A. W., \& Fomalont, E. B. 1996, ApJS, 105, 299

Fey, A. L., \& Charlot, P. 2000, ApJS, 128, 17

Fomalont, E. B., Frey, S., Paragi, Z., et al. 2000, ApJS, 131, 95

Gopal-Krishna, Patnaik, A. R., \& Steppe, H. 1983, A\&A, 123, 107

Gower, J. F. R., Scott, P. F., \& Wills, D. 1967, MmRAS, 71, 49

Griffith, M. R., Wright, A., Burke, B. F., \& Ekers, R. D. 1994, ApJS, 90, 179

Griffith, M. R., Wright, A., Burke, B. F., \& Ekers, R. D. 1995, ApJS, 97, 347

Hirabayashi, H., Fomalont, E. B., Horiuchi, S., et al. 2000, PASJ, 52, 997

Heidt, J., Tröller, M., Nilsson, K., et al. 2004, A\&A, 418, 813

Jauncey, D. L., Savage, A., Morabito, D. D., et al. 1989, AJ, 98, 54

Jauncey, D. L., King, E. A., Bignall, H. E., et al. 2003, PASA, 20, 151

Kameno, S., Sawada-Satoh, S., Inoue, M., Shen, Z.-Q., \& Wajima, K. 2001, PASJ, 53, 169

Katz, C. A., Moore, C. B., \& Hewitt, J. N. 1997, ApJ, 475, 512

Kedziora-Chudczer, L. L., Jauncey, D. L., Wieringa, M. H., Tzioumis, A. K., \& Reynolds, J. E. 2001, MNRAS, 325, 1411

Kellermann, K. I. 1966, Austr. J. Phys., 19, 195

Kesteven, M. J. L., Bridle, A. H., \& Brandie, G. W. 1977, AJ, 82, 541

King, E. A., McCulloch, P. M., Jauncey, D. L., et al. 1997, in Gigahertz peaked spectrum and compact steep spectrum radio sources, ed. I. A. G. Snellen, R. T. Schilizzi, H. J. A. Röttgering, \& M. N. Bremer, 17

Kovalev, Y. Y., Nizhelsky, N. A., Kovalev, Y. A., et al. 1999, A\&AS, 139,545

Kraus, J. D., Scheer, D. J., Dixon, R. S., Fitch, L. T., \& Andrew, B. H. 1968, ApJ, 152, L35

Kühr, H., Witzel, A., Pauliny-Toth, I. I. K., \& Nauber, U. 1981, A\&AS, 45, 367

Large, M. I., Mills, B. Y., Little, A. G., Crawford, D. F., \& Sutton, J. M. 1981, MNRAS, 194, 693

Lister, M. L., Kellermann, K. I., \& Pauliny-Toth, I. I. K. 2002, Proc. 6th European VLBI Network Symp., ed. E. Ros, R. W. Porcas, A. P. Lobanov, \& J. A. Zensus, 135

Lovell, J. E. J. 1997, Ph.D. Thesis, University of Tasmania
Lovell, J. E. J., King, E. A., Jauncey, D. L., et al. 2000, Adv. Space Res., 26, 715

Mantovani, F., Junor, W., Fanti, R., et al. 1992, MNRAS, 257, 353

Mantovani, F., Junor, W., Fanti, R., Padrielli, L., \& Saikia, D. J. 1997, A\&AS, 125,573

Mauch, T., Murphy, T., Buttery, H. J., et al. 2003, MNRAS, 342, 1117

O'Dea, C. P., Baum, S. A., \& Stanghellini, C. 1991, ApJ, 380, 66

O’Dea, C. P., \& Baum, S. A. 1997, AJ, 113, 148

O’Dea, C. P. 1998, PASP, 110, 493

Panajyan, V. G. 1998, Astrophysics, 41, 246

Peck, A. B., \& Taylor, G. B. 2000, ApJ, 534, 90

Perley, R. A. 1982, AJ, 87, 859

Shimmins, A. J., Searle, L., Andrew, B. H., \& Brandie, G. W. 1968 , ApJ, 1, L167

Slee, O. B. 1995, Australian J. Phys., 48, 143

Snellen, I. A. G., Schilizzi, R. T., Bremer, M. N., et al. 1999, MNRAS, 307, 149

Snellen, I. A. G., Schilizzi, R. T., \& van Langevelde, H. J. 2000, MNRAS, 319, 429

Snellen, I. A. G., Lehnert, M. D., Bremer, M. N., \& Schilizzi, R. T. 2002, MNRAS, 337, 981

Snellen, I. A. G., Mack, K.-H., Schilizzi, R. T., \& Tschager, W. 2003, PASA, 20, 38

Spoelstra, T. A. T., Patnaik, A. R., \& Gopal-Krishna 1985, A\&A, 152, 38

Stanghellini, C., O’Dea, C. P., \& Murphy, D. W. 1999, A\&AS, 134, 309

Stanghellini, C., Dallacasa, D., O’Dea, C. P., et al. 2001, A\&A, 377, 377

Taylor, G. B., Readhead, A. C. S., \& Pearson, T. J. 1996, ApJ, 463, 95 Taylor, G. B., \& Peck, A. B. 2003, ApJ, 597, 157

Tingay, S. J., Jauncey, D. L., Reynolds, J. E., et al. 1997, AJ, 113, 2025

Tingay, S. J., Reynolds, J. E., Tzioumis, A. K., et al. 2002, ApJS, 141, 311

Tingay, S. J., \& de Kool, M. 2003, AJ, 126, 723

Tingay, S. J., Edwards, P. G., \& Tzioumis, A. K. 2003a, MNRAS, 346, 327

Tingay, S. J., Jauncey, D. L., King, E. A., et al. 2003b, PASJ, 55, 351

Tornikoski, M., Lainela, M., \& Valtaoja, E. 2000, AJ, 120, 2278

Tornikoski, M., Jussila, I., Johansson, P., Lainela, M., \& Valtaoja, E. 2001, AJ, 121, 1306

Tzioumis, A. K., Jauncey, D. L., Preston, R. A., et al. 1989, AJ, 98, 36

Tzioumis, A. K., King, E. A., Reynolds, J. E., et al. 1998, in Radio emission from galactic and extragalactic compact sources, ed. J. A. Zensus, G. B. Taylor, \& J. M. Wrobel, ASP Conf. Ser., 144, 179

Ulvestad, J., Johnston, K., Perley, R., \& Fomalont, E. 1981, AJ, 86, 1010

Vermeulen, R. C., Ros, E., Kellermann, K. I., et al. 2003, A\&A, 401, 113

Wajima, K., Lovell, J. E. J., Kobayashi, H., et al. 2000, PASJ, 52, 329

Witt, H. J., Mao, S., \& Schechter, P. L. 1995, ApJ, 443, 18

Wright, A., \& Otrupcek, R. 1990, PKS Catalogue, Australia Telescope National Facility

Wright, A., Griffith, M. R., Burke, B. F., \& Ekers, R. D. 1994, ApJS, 91, 111

Wright, A., Griffith, M. R., Hunt, A. J., et al. 1996, ApJS, 103, 145 